\title{
INCB24360 Suppresses M1-like Macrophage Formation and NLRP3 Expression Whereas Increases IL-1 $\beta$ Secretion in RAW264.7 and BV-2
}

\section{Rong Ji}

Fudan University

Lixiang Ma

Fudan University

\section{Xinyu Chen}

Fudan University

Renqiang Sun

Fudan University

\section{Li Zhang}

Fudan University

Hexige Saiyin ( $\nabla$ saiyin@fudan.edu.cn )

Fudan University https://orcid.org/0000-0003-2993-6817

\section{Wenshi Wei}

Fudan University

\section{Research}

Keywords: macrophage, INCB24360, NLRP3, IL-1 $\beta$

Posted Date: December 23rd, 2020

DOI: https://doi.org/10.21203/rs.3.rs-131426/v1

License: (c) (1) This work is licensed under a Creative Commons Attribution 4.0 International License. Read Full License 


\section{Abstract}

Background: Macrophages switch between different functional phenotypes under the different physiological or path-physiological stimuli in tissue. Macrophage functional phenotype heavily affects disease progression, including inflammation, injury, neurodegenerative disease, and cancers. ID0-1, a druggable target, is an immunosuppressive enzyme expressed in tissue macrophages and induced by Interferon-y (IFN-ץ). How IDO-1 inhibitors affect the functional switches of macrophage are unknown.

Methods: IFN-y were used to increase ID 0-1 expression and 1-Methyl-D-tryptophan (1-MT) and INCB24360 (Epacadostat) were used to inhibit IDO-1 activity in RAW264.7 and BV-2. Western blotting, immunostaining and ELISA were used to evaluate protein expressions or secretion. RT-PCR were used to assess to transcription. TMR-Dextran and Latex beads were used to test endocytic, macropinocytic and phagocytic ability of macrophage and Fiji image and IMARIS were used to analyze images.

Results: Our results showed that INCB24360 preferentially suppresses the cellular size and filopodia growth and reduces fluid uptake, macropinocytosis, and phagocytic ability of IFN-y induced or noninduced RAW264.7 and BV-2 in vitro. 1-MT and INCB24360 suppress IFN-y induced or endogenous NLRP3 expression levels but not caspase-1 in RAW264.7 and BV-2. NLRP3 reduction induced by 1-MT and INCB24360 parallel with the decrease of NLRP3 gene transcription and an increase of IL-1 $\beta$ secretion.

Conclusions: Our data collectively showed that inhibiting IDO-1 with its inhibitors, especially INCB24360, preferentially suppresses cellular size and filopodia growth, NLRP3 expression, the endocytic, macropinocytic, and phagocytic capacity whereas increases IL-1 $\beta$ secretion in macrophage. Our findings do not rule out the roles of ID0-1 in the formation of M1-like macrophages, but they cast doubt on the robust anti-inflammatory effects of IDO inhibitors in the macrophage-mediated immune response.

\section{Introduction}

Macrophage, including tissue-resident macrophages or tumor-associated macrophages, is a vast immune cell population with significant heterogeneity, diversified origins, and different functions (1). Traditionally, macrophage polarizes to $\mathrm{M} 1$ or M2 macrophage under pathological and physiological stimuli in disease and health (2), and macrophage type significantly decides disease progression or outcome, including cancer and neurodegenerative disease et al. (2). Thus, the drugs that can polarize macrophages to a beneficial type are considered to modulate the disease progression and outcome (3).

Indoleamine 2,3-dioxygenase 1 (ID0-1), an immunosuppressive metabolic enzyme, is expressed in macrophages, dendritic cells, neoplastic cells, and placenta $(4,5)$. IDO-1 metabolizes tryptophan, which is the only precursor for the endogenous biosynthesis of nicotinamide adenine dinucleotide (NAD), into kynurenine (6). ID0-1 prevents the fetus's maternal T cell-driven immune rejection during pregnancy by depriving tryptophan and producing kynurenine (7). Excessive ID0-1 expression in neoplastic cells, macrophages, and dendritic cells causes tryptophan depletion in the milieu, and produces a large amount 
of kynurenine that suppresses T-cell proliferation and natural killer cells, promotes regulatory T-cell (Treg) development and myeloid-derived suppressor cells (MDSC), and prevents the overactivation of the immune response $(6,8,9)$. However, inhibiting IDO-1 in macrophage also surge the secretion of inflammatory cytokine in virus infection(10). Despite IDO-1 inhibitors' failure in cancer clinical trials (11), INCB24360, an IDO-1 inhibitor with strong Pharmacokinetic/pharmacodynamic (PK/PD) (12), and 1Methyl-D-tryptophan (1-MT), a substrate analog of d-tryptophan and I- tryptophan, is well-tolerated and safe in human clinical trials $(11,13)$. The response to inflammation and infection by cytokines, such as interferon-gamma (IFN- $\gamma$ ), increased IDO-1 expression in most cells, including macrophages (6). However, the endogenous biological effects of IDO-1 in macrophage activities are not well established. Overall, these features render ID0-1 inhibitors as an attractive molecule for regulating macrophage activities, thereby manipulating the trajectory of disease progression.

The M1 macrophage plays a proinflammatory role, while the M2 macrophage participates in the antiinflammatory process (2). M1 macrophage that responds to tissue insults and microbial infection is classically activated, increases iNOS, and secrete proinflammatory factors, such as TNF-a, IL-1 $\beta$, IL-6, superoxide, NO, ROS, and proteases (14-16). The release of proinflammatory cytokine by M1 macrophage and neurotoxic mediators induce a neurotoxicity effect on the brain and often create a vicious circle between dead neurons and acute inflammation (17). IL-4, IL-13, and IL-10 induced to form M2 macrophage, alternatively activated macrophage, and acquired inactivation status. M2 macrophage phagocytizes cellular debris and misfolded proteins, facilitate extracellular matrix (ECM) reconstruction and tissue repair, and support neuronal survival secreting neurotrophic factors $(14,18)$. The M2 macrophage can inhibit the proinflammatory immune response and express tissue repairing genes (18). However, macrophages in tissues do not often show a clear M1 or M2 phenotype in physiological or pathological conditions.

This study selected 1-MT and INCB24360 and tested the effects of 1-MT and INCB24360 on macrophage physiological activities. Here we show that INCB24360 preferentially and significantly inhibited M1-like macrophage activity and morphology, induced to form an M1-like macrophage with lower iNOS and TNF$a$ level whereas with increased IL-1 $\beta$ secretion. Also, we showed that 1-MT and INCB24360 treatment suppresses NLRP3 expression but not caspase-1.

\section{Materials And Methods}

\section{Cell line and drug treatment}

RAW264.7cells (Applied Biological Materials Inc.) and BV-2 cells (China Center for Type Culture Collection) were maintained in Dulbecco's Modified Eagle Medium (DMEM, Gibco, Carlsbad, CA, USA) with $10 \%$ fetal bovine serum (FBS, Gibco, Carlsbad, CA, USA) in a $37^{\circ} \mathrm{C} / 5 \% \mathrm{CO} 2$ circumstance. Mycoplasma free was demonstrated by Mycoplasma PCR Detection Kit (Biothrive, Myco-P-20, Shanghai, China). 
RAW264.7 and BV-2 cells were separately incubated with IFN-y, 1-MT, and INCB 24 hours. Inhibitions of IFN- $y$ induced IDO-1 are done as follow:

After incubating with IFN- $y$ for 12 hours, cells were directly added 1-MT/ INCB to the culturing medium without IFN- $\gamma$ withdrawal and then incubated for another 12 hours. RAW264.7 were separately incubated with MCC950 and Oridonin for 24 hours to inhibit NLRP3 inflammasome. The dosage of agents for

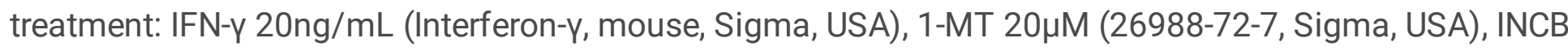

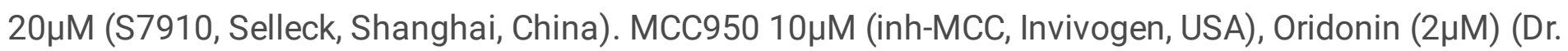
RB Zhou Hefei, China).

\section{Western Blot Analysis}

The cells were lysed in ice-cold RIPA buffer supplemented with phosphatase inhibitor PMSF 30min. The supernatant's protein concentration was measured by BCA assay kit (PD-BCA-125, Biothrive, Shanghai, China). $25 \mu$ g protein was loaded to $12 \%$ SDS-PAGE electrophoresis, and then transfer the protein from gel to PVDF membranes (Millipore, MA, USA). The membrane was blocked by $5 \%$ skim milk in TBST (PBS and $0.1 \%$ Tween), and then incubated $\left(4^{\circ} \mathrm{C}\right.$ overnight) with the antibodies. After finishing incubation in primary antibodies, the membrane was rinsed by $3 \times 5 \mathrm{~min}$ with TBST, then incubated with speciesspecific horseradish peroxidase-conjugated secondary antibodies (1:5000, Santa Cruz, Germany) for 60 min at room temperature. After 3x10 min washes with TBST, membranes were developed with supersensitive Enhanced Chemiluminescence substrate kit (Biothrive Ltd, ECL-P-100, Shanghai, China) for visualization by Tannon-4600.

The following antibodies were used in this work: Rabbit anti-iNOS (1:500, ab3523, Abcam, USA), rabbit anti-CD206(1:500, BM4881,Boster Biological Technology, CA, USA), rat anti-IDO(1:500, 654002, Biolegend, San Diego, CA, USA), mouse anti-NLRP3(1:1000, AG-20B-0014, Adipogen, CA, USA), rabbit anti-caspase1(1:500, 3866S, Cell Signaling Technology, MA, USA), rabbit-anti-S6K(R\&D Systems, MN, USA), rabbit antip-S6K(R\&D Systems, MN, USA), anti-a-tubulin (1:10000, HRP-66031,Proteintech, Wuhan, China), and antiGAPDH (1:10000, HRP-60004,Proteintech, Wuhan, China).

\section{Real-time PCR analysis}

EZ-press RNA Purification Kit (B0004DP, USA) was used to extract total RNA by following the manufacturer's protocol. Verso cDNA kit (Thermo Scientific, Lafayette, CO, USA) is applied to cDNA reverse transcription. Quantitative real-time PCR was performed on a Bio-Rad Cx96 Detection System (Bio-Rad, USA) using an SYBR green PCR kit (Applied Biosystems, USA). Primers: 1 ; iNOS, forward primer (5'-3')GGAGTGACGGCAAACATGACT, reverse primer(5'-3')TCGATGCACAACTGGGTGAAC; 2; CD206, forward primer (5'-3')CTCAACCCAAGGGCTCTTCTAA, reverse primer(5'-3')AGGTGGCCTCTTGAGGTATGTG; 3; TNF-a, forward primer (5'-3')CTGTGAAGGGAATGGGTGTT, reverse primer(5'-3')GGTCACTGTCCCAGCATCTT; 4; Arg-1, forward primer(5'-3')CTCCAAGCCAAAGTCCTTAGAG, reverse primer(5'-3')GGAGCTGTCATTAGGGACATCA; 5; NLRP3, forward primer(5'-3')ATGCTGGCTTCGACATCTCCT, reverse 
primer(5'-3')GTTTCTGGAGGTTGCAGAGC; 6; Caspase-1, forward

primer(5'-3')AGATGCCCACTGCTGATAGG, reverse primer(5'-3')TTGGCACGATTCTCAGCATA; 7; GAPDH, forward primer(5'-3')ATACGGCTACAGCAACAGGG, reverse primer(5'-3')GCCTCTCTTGCTCAGTGTCC.

\section{Immunofluorescence staining}

Immunostaining was done as previously described (19). Briefly, cells were fixed by 4\% PFA, incubated with primary antibody overnight, and incubated with fluorescent secondary antibodies with DAPI for 1 hour. After staining, slides were mounted by mounting medium for microscopy. The following antibodies were used: rabbit anti-iNOS (1:200, ab3523, Abcam, USA), rabbit anti-CD206 (1:200, BM4881, Boster Biological Technology, CA, USA), rat anti-F4/80(1:100, ab16911, Abcam, USA), mouse anti-NLRP3(1:400, AG-20B-0014, Adipogen, CA, USA). 4',6-diamidino-2-phenylindole (DAPI, 1:2000, D9542, Sigma, MO, USA). Images were obtained using a Leica SP8 confocal microscope (Leica Microsystems, Japan). Cell counts and morphological analyses were performed using Fiji (ImageJ) software.

\section{ELISA Assay}

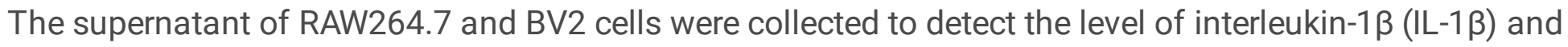
interleukin-18 (IL-18) with mice enzyme-linked immunosorbent assay kit (MeiLian, Shanghai, China) according to the instructions of the provider.

\section{Transwell Migration Assay}

Cells were resuspended in serum-free DMEM, and plate $150 \mu \mathrm{l}(\mathrm{E} 5 / \mathrm{ml} \|$ cell suspension into the upper chamber of the Transwell. After that, $800 \mu$ of DMEM containing 10\% FBS were added to the lower chamber. After incubating of RAW264.7 for $48 \mathrm{~h}$ or BV-2 for $24 \mathrm{~h}$, the upper chambers were removed, aspirated the culture solution in an upper chamber, fixed the chambers with 4\% PFA for 15 minutes, washed the chambers three times with PBS, and then stained the chambers in the crystalline violet solution for 30 minutes. After staining, the upper layer of cells gently swabbed with a cotton swab moistened with PBS, rinsed with PBS three times, and dried for microscopy.

\section{Edu Assay}

Edu assay (Invitrogen)was done by following the supplier protocol. After drug treatments, an Edu working solution (10mM, 1:2000, with green fluorescence) was added to the medium and then incubated for another $12 \mathrm{~h}$. After incubation, the cells were fixed by $4 \%$ PFA for 20 min, rinsed with PBS, permeabilized by $0.2 \%$ Triton $\mathrm{X}-100$ for $20 \mathrm{~min}$, and then added freshly-prepared Click-iT ${ }^{\mathrm{TM}}$ reaction solution and incubated for 30 minutes. After washing, the cell culturing slides were mounted to a slide for microscopy.

\section{Imaging and Image analysis}

Leica SP8 microscopy was used to scan all images. Fiji (ImageJ) software was used to count cell and morphological analyses. IMARIS9.5 was used for particle size analysis. 


\section{Statistics}

SPSS20.0 and GraphPad were used for data analysis.

\section{Results}

\section{INCB24360 reduces iNOS expression in RAW264.7 and BV-2}

IFN-y polarizes macrophage to M1 type, increases ID0-1 expression (20). To test the ID0-1 inhibitor's effect on the macrophage polarization, we have treated RAW264.7 and BV-2 with IFN- $\gamma$ at $20 \mathrm{ng} / \mathrm{mL}$ to

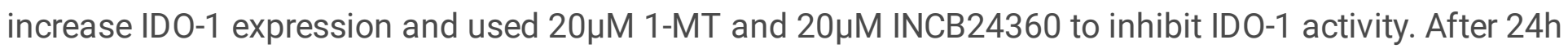
treatment, we have detected CD206, an M2 marker, iNOS, an M1 marker, IDO-1 by western blotting. Consistent with previous findings $(21,22)$, we found that IFN- $y$ treatment increased IDO-1 and iNOS expression in RAW264.7 and BV-2 (Fig.1A, B, C, Si-Fig.1A). INCB24360 treatment reduced IDO-1 expression in RAW264.7 but not in BV-2 (Fig.1B, Si-Fig.1A), INCB24360 treatment reduced iNOS expression in RAW264.7 and BV-2(Fig.1C, Si-Fig.1A). The decreases of iNOS induced by INCB24360 but not 1-MT treatment are statistically significant in RAW264.7 (Fig.1C). 1-MT and INCB24360 treatment did not affect CD206 expression in RAW264.7 (Fig.1D). We also detected the transcription of iNOS, TNF-a, CD206, and Arginase 1 (Arg1) genes by RT-PCR. Our data showed that INCB24360 significantly decreased the transcriptions of iNOS, TNF- $a$ in RAW264.7 and BV-2 compared to the control and IFN- $y$ treated RAW264.7 (Fig.1E, F) and BV-2 (Si-Fig.1B, C); 1-MT treatment only decreased the transcriptions of iNOS, TNF-a in BV2 cells but not in RAW264.7 (Fig.1E, F, Si-Fig.1B, C). The CD206 gene transcriptions were increased in INCB24360 treated RAW264.7 and BV-2 compared to the control group (Fig.1G, Si-Fig.1D); The transcriptions of Arg 1 gene also were increased in INCB24360 treated RAW264.7 but not BV-2 (Fig.1H, Si-Fig.1E). However, 1-MT treatment did not change the transcriptions of Arg 1 gene in RAW264.7 and BV-2 compared to the control group (Fig.1H, Si-Fig.1E).

Consistent with western blotting, immunofluorescent staining of RAW264.7 and BV-2 treated with INCB24360 and I-MT showed that iNOS expression significantly decreased INCB24360 groups but not CD206 compared to the control group (Fig.1I-K, Si-Fig.1F-H). The data indicate that INCB24360 might preferentially inhibit M1-like RAW264.7 and BV-2 formation.

\section{INCB24360 suppresses the increase of cellular body and membrane filopodia formation of RAW264.7 and BV-2}

The cellular morphological changes were correlated with different functional status (23). Non-polarized RAW264.7 is a small and round cell with few processes (20). If polarized, RAW264.7cells form a large round pancake-like cell, representing $M 1$, or a slimmer cell with longer processes, representing the M2 stage (20). To see whether the decrease of iNOS expression in RAW264.7 and BV-2 after INCB24360 treatment parallel with the cellular morphological changes and filopodia formation, we have assessed the morphology of RAW264.7 and BV-2 after IFN-y, 1-MT, and INCB24360 treatment (Fig.2A, Si-Fig. 2A). IFN-y treatment dramatically increased the total polarized RAW264.7 and BV-2, while 1-MT or INCB24360 
treatment did not significantly change the total polarized RAW264.7 count (Fig.2B) and BV-2 (Data not shown). We divided the polarized macrophage into M1 or M2 macrophage based on their morphology. Consistent with other observations(20), our results showed that IFN- $y$ treatment preferentially increased M1 RAW264.7 and BV-2 and slightly decreased M2 macrophage(Fig.2C, D, Si-Fig. 2B, C); INCB24360 treatment significantly decreased the proportion of M1 RAW264.7 and BV-2 compared to the control and IFN-y treated group (Fig.2C, Si-Fig.2B). 1-MT treatment did not change the proportion of M1 and M2 in RAW264.7 (Fig.2C, D). However, both 1-MT and INCB24360 slightly increased the proportion of M2 BV-2 compared to the control group (Si-Fig.2C). These observations are consistent with the formation of an M1-like macrophage.

M1 macrophage showed multiple filopodia that facilitate the phagocytizing of fluid or foreign substances, including inorganic particles (24). We have treated RAW264.7 and BV-2 cells with IFN- $\gamma, 1-M T$, and INCB24360 for 24h, fixed the cells and stained with phalloidin Alexa-488, an actin-binding dye, and DAPI. We have scanned cells with high-resolution confocal microscopy and observed that macrophages induced by IFN- $\gamma$ formed a ruffled border decorated with abundant filopodia (Fig.2E, Si-Fig.2D). Some RAW264.7 in IFN-y treated group formed multi-nucleus cells that resemble osteoclasts (Data not shown). INCB24360 treated macrophages showed fewer filopodia than the control, IFN-y and 1-MT groups in RAW264.7 and BV-2 (Fig.2E, Si-Fig.2D). We further measured the cellular perimeter and counted the density of filopodia on the cellular membrane. The data showed that 1-MT and INCB24360 treatment did not change the cellular perimeter and decreased filopodia density on the cellular membrane (Fig.2F, G, Si-

Fig 2E, F). Collectively, these findings implied that INCB24360 and 1-MT preferentially block the formation of M1-like macrophages.

\section{INCB24360 reduces the TMR-dextran uptake, macropinocytosis, and phagocytic capacity of RAW264.7 and BV-2}

To see the decrease of iNOS expression and reduction of filopodia and ruffled border by INCB24360 treatment also decrease the phagocytic ability in RAW264.7 and BV-2, we have administrated TMRdextran into RAW264.7 and BV-2 after treating IFN-y, 1-MT, and INCB24360 for 24h. After 1 hour of TMRdextran treatment, we fixed the cells and counter-stained by phalloidin-Alex-488 and DAPI. After scanning under microscopy with Z-stack, we have analyzed the total amount of dextran in four group cells by ImageJ. The results showed that INCB24360 treatment significantly reduced DTR-dextran uptake compared to the control and IFN- $y$ treated group in RAW264.7 but not in BV-2 (Fig.3A, 3B, 3D, 3E).

Macropinocytosis is a way by which macrophage uptakes large amounts of extracellular fluid (25). Macropinosome formation was dependent on membrane ruffling. The decrease of filopodia on the ruffled border after the INCB24360 treatment implied that INCB24360 might reduce the macropinocytosis of RAW264.7 and BV-2. The macropinosomes' size is heterogeneous; the diameter range from $0.2 \mu \mathrm{m}$ to $5 \mu \mathrm{m}$ (26). The vesicles, which are larger than $0.75 \mu \mathrm{m}$, were defined as apparent macropinocytic vesicles in DTR-dextran uptake assays by some researchers (27). We have analyzed the macropinocytic vesicles 
that were larger than $0.75 \mu \mathrm{m}$ by Imaris9.6 software and found that INCB24360 treatment decreased the number of phagocytic vesicles larger than $0.75 \mu \mathrm{m}$ in RAW264.7 and BV-2 (Fig.3A, C, D, F).

Macrophage, especially tissue-resident macrophage, can also phagocytize foreign-derived particulates such as alum, silica et al. M1 macrophage can phagocytize foreign-derived particulates $(28,29)$. We also treated RAW264.7 and BV-2 with Latex beads, a spherical polymer particle, fluorescence red, and $1 \mu \mathrm{m}$, after treating with IFN- $y, 1-M T$ and INCB24360 for 24h. After fixing with 4\% PFA, we analyzed the phagocytized Latex beads in both cells and found that RAW264.7 (Data not shown) has a weaker ability to phagocytize Latex beads compared to BV-2. We observed that 1-MT and INCB24360 treatment slightly decrease the Latex beads uptake of BV-2 compared to the control, but the decreases are not statistically significant (Fig.3G-J).

Contrary to M2, M1 is weak in motility $(30,31)$. We also test 1-MT and INCB24360 effects on the migration and proliferation of macrophages; we have used the Transwell Migration Assay to test the migrating ability and Edu staining to test the proliferative potential. Consistent with the formation of M1 macrophage, the results showed that IFN- $\gamma$ dramatically decreases the migration ability of RAW264.7 and BV-2(Si-Fig.3A-C) but not effects the proliferation of RAW264.7 and BV-2(Si-Fig.3D-F); 1-MT and INCB24360 do not affect the migration and proliferation of RAW264.7 and BV-2 (Si-Fig.3). Taken together, 1-MT and INCB24360, especially INCB24360, preferentially inhibit the phagocytic ability and macropinocytosis of the M1-like macrophage but not migrating and proliferating capacity.

\section{INCB24360 suppresses IFN-y-induced iNOS upregulation in RAW264.7}

INCB24360 significantly blocks M1 RAW264.7 and BV-2 formation. IFN-y increased ID0-1 expression (9). Thus, we hypothesized that INCB24360 might suppress IFN-y induced iNOS increase. To test if 1-MT and INCB24360 suppress IFN- $\gamma$-induced iNOS upregulation, we treated RAW264.7 and BV-2 with IFN- $\gamma$ for 12h, and then added 1-MT and INCB24360 for 24h. We have detected the expression CD206, iNOS, and IDO-1. Both 1-MT and INCB24360 treatment in the IFN-y treated group significantly decreased iNOS levels but not changed CD206 levels compared to the IFN-y treatment group in RAW264.7 (Fig.4A-C); more decreases of iNOS were observed in the INCB24360 group (Fig.4B); 1-MT and INCB24360 did not change iNOS and CD206 expression in IFN-y induced BV-2 (Si-Fig.4A). We also detected the transcription of iNOS, TNF-a, CD206, and Arg1 genes by RT-PCR in four group cells. Our data showed that INCB24360 treatment suppressed the transcriptions of iNOS, TNF- $a$ in IFN- $\gamma$ induced RAW264.7 and BV-2 (Fig.4E, F, Si-Fig.4B, C); INCB24360 treatment significantly increased the transcriptions of CD206 but not Arg1 genes in IFN- $\gamma$ treated RAW264.7 (Fig.4H), 1-MT treatment does not significantly affect the transcription of iNOS, TNF-a, CD206, and Arg1 genes in IFN-y treated RAW264.7(Fig.4E-H). In agreement with western blotting results, immunofluorescent staining of results showed that iNOS expression significantly decreased in IFN- $\gamma$ induced cells treated with INCB24360 or 1-MT compared to the IFN- $\gamma$ treatment RAW264.7 and BV-

2(Fig.4l, J, Si-Fig.4D, E). 1-MT treatment significantly increased CD206 expression in IFN-y induced RAW264.7, 1-MT and INCB24360 increased CD206 expression in IFN-y induced BV-2 (Fig.4I, K, Si-Fig.4D, F). 
To test if INCB24360 also inhibits IFN-y-induced cellular size and filopodia growth, we treated RAW264.7 and BV-2 with IFN- $y$ for $12 \mathrm{~h}$ and then added 1-MT or INCB24360 for $24 \mathrm{~h}$. After imaging, we divided the macrophage into M1 or M2 based on macrophage morphology and found that INCB24360 treatment not only decreased the total polarized RAW264.7(Fig.5A, B) and BV-2(Data not shown) driven by IFN- $\gamma$ but INCB24360 treatment preferentially decreased IFN- $\gamma$-induced M1 RAW264.7 and BV-2 (Fig.5A, C, Si-Fig.5A, B). Interestingly, INCB24360 treatment significantly increased IFN-y-induced M2 macrophage proportion

(Fig.5A, D, Si-Fig.5A, C). 1-MT treatment decreased the count of total IFN- $\gamma$-induced polarized macrophage, and this decrease did not have a preference for M1 or M2 (Fig.5A-D, Si-Fig.5A-C). The findings further support that INCB24360 can reverse IFN-y driven M1 macrophage formation.

To test if ID 0-1 inhibitors block IFN-y-driven ruffled border formation and filopodia growth, we treated RAW264.7 and BV-2 with IFN-y for 12h, and then added 1-MT or INCB24360 for 24h, and fixed the cells, and stained with phalloidin Alexa-488 and DAPI. We also scanned the cells with confocal microscopy with Z-stack, analyzed cellular perimeters, and filopodia density on the cellular border. Our data showed that INCB24360 inhibits IFN-y induced ruffled border and filopodia formation in RAW264.7 and BV-2

(Fig.5E, Si-Fig.5D). The measuring and counting data showed that both 1-MT and INCB24360 treatment significantly inhibited the increase of cellular size induced by IFN-y in RAW264.7 (Fig.5F) but not in BV-2 (Si-Fig.5E); INCB24360 treatment also inhibits the increase of filopodia density on the cellular membrane induced by IFN-y (Fig.5G, Si-Fig.5F); 1-MT treatment does not restrict the increase of filopodia density on the cellular membrane induced by IFN-y (Fig.5G, Si-Fig.5F). Our data showed that INCB24360 inhibits IDO1 induced cellular size increase and filopodia formation. 1-MT weakly inhibits ID0-1-induced cellular size increase.

\section{INCB24360 suppress IFN-y induced endocytic, macropinocytic phagocytic ability}

IFN- $y$ increased the phagocytic ability of RAW264.7 and BV-2 (Fig.3A, D). 1-MT and INCB24360 reduce the endocytic, phagocytic, and macropinocytic abilities of RAW264.7 and BV-2. We hypothesized that 1-MT and INCB24360 can decrease the endocytic and macropinocytic ability induced by IFN- $y$. To test this hypothesis, we have treated BV-2 and RAW264.7 with IFN-y for 12 hours and then treated with 1-MT and INCB24360 for another 12 hours. After that, we have administrated DTR-dextran to the culturing medium. After treating with DTR-dextran for $1 \mathrm{~h}$, we have fixed the cells and counter-stained by phalloidin Alex-488. After scanning under microscopy with Z-stack, we have analyzed the total amount of dextran in four group cells. The results showed that INCB24360 decreased TMR-dextran uptake ability in both RAW264.7 and BV-2; 1-MT significantly decreased TMR-dextran uptake ability in RAW264.7 but not in BV-2 (Fig.6A, $B, D, E)$. We have analyzed the DTR-dextran phagocytic vesicles that were larger than $0.75 \mu \mathrm{m}$, which is reflective of macropinocytosis, by Imaris9.6 software and found that INCB24360 treatment decreased the number of phagocytic vesicles larger than $0.75 \mu \mathrm{m}$ in RAW264.7 and BV-2, and 1-MT significantly decreased the number of phagocytic vesicles larger than $0.75 \mu \mathrm{m}$ in RAW264.7 but not in BV-2 (Fig.6A, C, D, F). 
To test the effect of 1-MT and INCB24360 on macrophage phagocytic ability, we treated RAW264.7 and BV-2 with IFN- $y$ for $12 \mathrm{~h}$, and then added 1-MT or INCB24360 for $24 \mathrm{~h}$, and added Latex beads to the culturing medium to phagocytize. After fixing, we analyzed the phagocytized Latex beads in the two cells and found that INCB24360 and 1-MT treatment significantly decreased the count of BV-2 that phagocytize Latex beads, the number of phagocytic latex beads in each IFN- $\gamma$ induced BV-2 treated with INCB24360 was significantly lower than that in IFN- $y$ induced BV-2, the number of phagocytic latex beads in each IFN-y induced BV-2 treated with 1-MT was not significantly lower than that in IFN- $\gamma$ induced BV-2 (Fig.6G-J). Collectively, I-MT and INCB24360, especially INCB24360, preferentially inhibit the endocytic, phagocytic, and macropinocytic ability of M1-like macrophages induced by IFN-ץ.

\section{1-MT and INCB24360 reduced NLRP3 expression and NLRP3 gene transcription in RAW264.7 and BV-2}

NLRP3 inflammasome, a large protein complex, includes NLRP3, ASC, and caspase 1 (32). NLRP3 inflammasome is involved in M1 macrophage formation and increases IL-1 $\beta$ production in inflammation (33). Curcumin, an IDO-1 inhibitor, inhibits chronic unpredictable mild stress (CUMS) induced NLRP3 expression, reduces depressive-like behaviors in rats (34). Thus, we hypothesized that 1-MT and INCB24360 might also suppress NLRP3 expression. To test if the inhibition of ID0-1 with 1-MT and INCB24360 reduce NLRP3 expression, we have treated RAW264.7 and BV-2 with IFN-y, 1-MT, and INCB24360 for 24h and detected NLRP3 expression by western blotting, and found that both 1-MT and INCB24360 decreased NLRP3 expression in RAW264.7 and BV2 but not caspase-1 (Fig.7A), the decreases of NLRP3 expression in INCB24360 group are significant (Fig.7B), the reduction of NLRP3 expression in

the 1-MT group is statistically non-significant (Fig.7B). We also detected NLRP3 and Caspase-1 gene transcription by RT-PCR. The data showed that INCB24360 reduced the transcription of the NLRP3 gene but not Caspase-1 (Fig.7D, E, Si-Fig-6B, C). We also used immunostaining with NLRP3 and iNOS antibodies to detect the changes in NLRP3 and iNOS expression. Consistent with immunoblotting and RTPCR results, INCB24360 treatment significantly decreases NLRP3 expression in RAW264.7 (Fig.7F, G) and BV-2(Si-Fig.6D, E).

To test if 1-MT and INCB24360 also can decrease IFN-y induced NLRP3 expression, we also treated RAW264.7 with IFN-y for 12h, and then added 1-MT or INCB24360 for 24h to inhibit IDO-1 activity. Our data showed that both 1-MT and INCB24360 could inhibit IFN-y induced NLRP3 expression but not caspase-1 (Fig.7I-K). The RT-PCR data also showed that INCB24360 and 1-MT reduced IFN-y induced NLRP3 gene transcription in both RAW264.7 and BV-2 but not caspase-7(Fig.7L, M, Si-Fig.6H, I). We have immunostained those cells with NLRP3 and iNOS antibodies, and immunofluorescent staining results in RAW264.7 are consistent with protein blotting results (Fig.7N-P, Si-Fig.6J-L).

\section{1-MT and INCB24360 treatment moderately increase IL-1 $\beta$ secretion in RAW264.7 and BV-2}

NLRP3 inflammasome activates caspase 1 that cleaves pro-IL-1 $\beta$ and pro- IL-18 and secretes IL-1 $\beta$ and IL-18 (32). Inhibiting IDO with 1-MT dramatically surge the proinflammatory cytokine secretion of macrophage in influenza virus infection, including IFN- $\gamma$, IL-1 $\beta$, IL-6, and TNF- $a$ (10). To test how the modest decrease of NLRP3 expression by 1-MT and INCB24360 treatment effects IL-1 $\beta$ and IL-18 


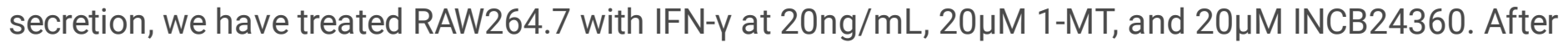
treating for $24 \mathrm{~h}$, we have detected IL-1 $\beta$ and IL-18 secretion levels in the medium by ELISA. Our results showed that IL-1 $\beta$ and IL-18 levels in the culturing medium of IFN- $\gamma, 1-M T$, and INCB24360 treated RAW264.7 and BV-2 significantly increased compared to the control (Fig.8A, B, Si-Fig.7A, B). It implied that endogenous IDO-1 might can prevent the overactivation of macrophages.

Mammalian Target of Rapamycin (mTORC1) is a nutrient-dependent kinase regulating cellular growth and metabolism (35). Tryptophan deprivation by IFN-y induced IDO-1 upregulation inhibits mTORC1 kinase, and tryptophan or 1-MT treatment reversed IDO-1 mediated inhibition of mTORC1 kinase; mTORC1 stimulation by 1-MT relies specifically on tryptophan depletion $(36,37)$. mTORC1 inhibition also decreased glycolysis and suppressed both LPS and ATP induced pro-IL-1 $\beta$ maturation and caspase-1 activation in macrophages (38). Thus, we hypothesized that INCB24360 treatment reduced tryptophan deprivation induced by IFN-y in the milieu, consequently activating mTORC1. We have used to a phosphorylated (p) S6K (T389) antibody to evaluate mTORC1 activation levels in RAW264.7.

Interestingly, we did not observe a direct effect of 1-MT and INCB24630 treatment on pS6K (T389) levels in RAW264.7 (Fig.8C). We observed that inhibiting IFN-y induced IDO-1 with 1-MT and INCB24360 increases IL-1 $\beta$ and IL-18 secretion in RAW264.7 and BV-2(Fig. 8D, E, Si-Fig.7C, D). The increase of IL-1 $\beta$ secretion in INCB24360 treated RAW264.7 and BV-2 induced by IFN- $y$ were significantly higher than that in RAW264.7 and BV-2 induced by IFN-y alone (Fig. 8D, Si-Fig.7C). Interestingly, 1-MT significantly increased IL-18 secretion in RAW2 and BV-2 induced by IFN-y but not IL-1 $\beta$ (Fig. 8D, E, Si-Fig.7C, D). Also, in agreement with other findings (36), inhibiting IFN-y induced RAW264.7 with 1-MT and INCB24360 dramatically increased in pS6K (T389) levels (Fig. 8F). Activation of mTORC1 by 1-MT and INCB24360 in IFN- $\gamma$ induced might partially explain why 1-MT and INCB24360 increase IL-1 $\beta$ secretion in RAW264.7.

Curcumin inhibits CUMS-induced NLRP3 expression (34). NLRP3 deficiency also inhibits the LPS-induced IDO increases in the hippocampal microglia (39). These data indicate that IDO-1 and NLRP3 regulated each other in macrophages. Thus, we hypothesized that inhibiting NLRP3 by its inhibitors might affect ID0-1 expression in macrophages. To test if NLRP3 inhibition affects endogenous ID0-1 levels in RAW264.7, we have inhibited NLRP3 in RAW264.7 with MCC950, an inhibitor of NLRP3 targeting its ATPhydrolysis (40), and Oridonin, a covalent NLRP3 inhibitor (41). After exposing RAW264.7 to MCC950 and Oridonin for 24h, we have detected NLRP3 and IDO-1 expression (Fig. 8G). Our western blotting and immunofluorescent staining data showed that inhibiting NLRP3 with Oridonin and MCC950 increased IDO-1 expression in RAW264.7 (Fig. 8G-J). Our data indicate that NLRP3 and IDO-1 regulate each other in macrophages.

\section{Discussion}

Millions of versatile macrophages reside in the human organs and solid tumors (1). Its phenotype switches control macrophage physiological and pathological action (2). Thus, the functional switches of macrophages dominantly affect the disease's outcome $(1,2)$. ID 0-1, an immunosuppressive enzyme that represses $T$ cell activities in cancer and fetus and restricts the overactivation of the immune response in 
inflammation $(42,43)$. Using 1-MT and INCB24360, we found that INCB24360 could restrict the cellular size and filopodia growth and reduce their endocytic, phagocytic, and macropinocytic capacity in RAW264.7 and BV-2. 1-MT and INCB24360 treatment also reduced NLRP3 expression in RAW264.7, whereas increasing the secretion of IL-1 $\beta$. Our finding implied that manipulating endogenous or induced ID0-1 in macrophages with INCB24360 induced to form of a specific macrophage with lower endocytic, phagocytic, and macropinocytic capacity and possesses lower NLRP3 transcription, whereas higher IL-1 $\beta$ secretion. Our study revealed the paradox roles of endogenous and induced ID 0-1 in macrophage activities, including tissue-resident macrophages and monocyte-derived macrophages.

Macrophages, including tissue-resident or tumor-associated macrophages, are the most abundant phagocytic cells in murine and human tissues, which connect innate and adapt immune response (1). Macrophages are versatile cells that are generally divided into M1 and M2 types for the sake of simplicity. However, macrophage classification into M1 and M2 over-simplified macrophages' versatile roles in tissues and is too bipolar (18). IDO-1 is upregulated in M1 macrophage. Our data showed that INCB24360 but not 1-MT significantly suppresses iNOS expression and TNF- $a$ in both RAW264.7, a monocyte-derived macrophage, and BV-2, microglia.

Nevertheless, INCB24360 does not affect M2 cell marker-CD206 and Arg 1 expression. Consistent with the decrease of ruffled border and filopodia, INCB24360 treatment significantly decreased the endocytic, macropinocytic, and phagocytic ability of INCB24360 treated macrophages. The decrease of endocytic,

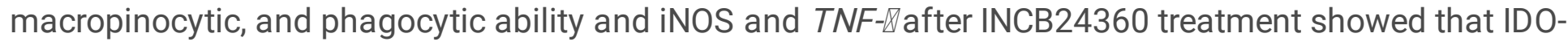
1 drive to form a conventional M1-like macrophage formation. IDO-1 is an immunosuppressive molecule; 1-MT treatment dramatically surges IL-1 $\beta$ secretion in macrophages in virus inflammation (10). The enhancement of IL-1 $\beta$ secretion after INCB24360 treatment is not consistent with the formation of a typical M1 macrophage. IL-1 $\beta$ is a critical proinflammatory cytokine in the immune response(44). Our findings suggest that endogenous or induced IDO-1 in macrophages might prevent the overactivation of macrophage-mediated immune response by limiting IL-1 $\beta$ secretion. Another concern is that tryptophan also is the only source of nicotinic acid (45). IDO-1 is the first rate-limiting enzyme that converts tryptophan into kynurenines that metabolize into nicotinic acid (45). The administration of nicotinic acid and its metabolites suppress IL-1 $\beta$ secretion in macrophage (46-48). If INCB24360 or 1-MT treatment reduces nicotinic acid deficiency in cells, which increases IL-1 $\beta$ secretion, it needs to be addressed in the future.

Previous studies showed that IDO-1 is a downstream enzyme of the NLRP3 inflammasome, and IDO-1 upregulation induced by lipopolysaccharide (LPS) is diminished in the glial cells of $\mathrm{N} / r p 3^{-/-}$mouse, including microglia $(39,49)$. Inhibition of IDO-1 with curcumin decreased NLRP3 expression (34). We showed that both 1-MT and INCB24360 treatment reduced endogenous or IFN- $\gamma$ induced NLRP3 expression and reduced expression by inhibiting NLRP3 gene transcription. However, inhibiting NLRP3 with its inhibitors also increased IDO-1 expression. These data support that NLRP3 and ID0-1 regulate each other, and the interaction might depend on external stimuli or the cellular polarization status. The failure of INCB24360 in cancer clinical trials renders us the complexity of ID0-1 roles in the immune 
system (50). The differences of 1-MT and INCB24360 in inhibiting ID0-1 activity might partially explain the differences between the two drugs on macrophage polarization. Treg and CD8 $+T$ cells population changes in tumor milieu are used to interpret the effectiveness of IDO-1 inhibitors in cancer treatment (11, 37). The macrophage population prevails over other immune cells in human organs and solid cancers. However, macrophage activities are often neglected in IDO inhibitor treatment. IL-1 $\beta$ is a proinflammatory cytokine, enhances the inflammatory reaction. Based on the increase of IL-1 $\beta$ in macrophages after treating with 1-MT and INCB24360, it is worth evaluating macrophage and neutrophils infiltration after IDO-1 inhibitors treatment. In addition, we observed that inhibiting IDO-1 with 1-MT and INCB24360 in IFN- $\gamma$ induced cells not decreased IL- $1 \beta$ secretion and preferentially increased mTORC1 activity, which is measured by the pS6K antibody. This scenario was not seen in non-1-MT and INCB24360 RAW264.7. This observation reflected that macrophage in different status reacts differently to IDO-1 inhibitors.

Collectively, our study revealed that INCB24360 significantly inhibits the cellular size and filopodia

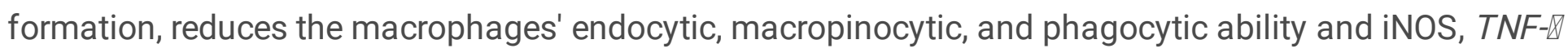
and NLRP3 expression whereas upregulates IL-1ß secretion in macrophage in vitro, implicating that INCB24360 could manipulate macrophage activities and ID0-1 might have a capacity to prevent overactivation of macrophage in immune response.

\section{Conclusion}

We selected 1-MT and INCB24360 and tested the effects of 1-MT and INCB24360 on macrophage physiological immunological activities. our data revealed that INCB24360 significantly inhibits the cellular size and filopodia formation, reduces the macrophages' endocytic, macropinocytic, and phagocytic ability and iNOS, TNF- $\triangle$ and NLRP3 expression whereas upregulates IL-1 $\beta$ secretion in macrophage in vitro, implicating that INCB24360 could manipulate macrophage activities and IDO-1 might have a capacity to prevent overactivation of macrophage in immune response. Our findings do not rule out the roles of ID0-1 in the formation of M1-like macrophages, but they cast doubt on the robust anti-inflammatory effects of IDO inhibitors in the macrophage-mediated immune response.

\section{Abbreviations}




\begin{tabular}{|ll|}
\hline IDO-1 & Indoleamine 2,3-dioxygenase 1 \\
\hline INCB & INCB24360 \\
\hline 1-MT & 1-Methyl-D-tryptophan \\
\hline NAD & Nicotinamide adenine dinucleotide \\
\hline Treg & Regulatory T-cell \\
\hline MDSC & Myeloid-derived suppressor cells \\
\hline PD & Pharmacokinetic \\
\hline IFN-Y & Pharmacodynamic \\
\hline TNF-a & Tumor necrosis factor-alpha \\
\hline IL-1 $\beta$ & Interleukin-1 $\beta$ \\
\hline iNOS & Inducible nitric oxide synthase \\
\hline IL-6 & Interleukin-6 \\
\hline IL-4 & Interleukin-4 \\
\hline IL-13 & Interleukin-13 \\
\hline IL-10 & Interleukin-10 \\
\hline ECM & Extracellular matrix \\
\hline ROS & Reactive oxygen species \\
\hline Edu & 5-Ethynyl-2' -deoxyuridine \\
\hline ELISA & Enzyme-linked immunosorbent assay \\
\hline CD206 & mannose receptor (Cluster of Differentiation 206) \\
\hline Arg1 & Arginase-1 \\
\hline NLRP3 & NLR family pyrin domain containing 3 \\
\hline ASC & Apoptosis-associated speck-like protein containing a CARD \\
\hline CUMS & chronic unpredictable mild stress \\
\hline
\end{tabular}

\section{Declarations}


Acknowledgements

We thank all people who support this project and open their facility to finish this work during the Covid-19 season.

\section{Authors' contributions}

Conceptualization, H.S., R.J.;

Methodology, H.S., R.J., and RQ, S.;

Investigation, H.S., R.J., LX, M., XY, C.;

Writing - Original Draft, H.S. and R.J.;

Funding Acquisition, LX, M., H.S., L.Z. and WS, W.;

Resources, LX, M., H.S., and WS, W.;

Supervision, H.S., L.Z. and WS, W.

\section{Funding}

This study was supported by funds from National Natural Science Foundation of China (81371220).

Availability of data and materials

All data generated or analyzed during this study are included in this published article [and its supplementary material file].

\section{Ethics approval and consent to participate}

Not applicable.

\section{Consent for publication}

Not applicable.

\section{Declaration of interests}

The authors declare no competing interests.

\section{References}

1. Davies LC, Jenkins SJ, Allen JE, Taylor PR. Tissue-resident macrophages. Nat Immunol. 2013;14(10):986-95. 
2. Murray PJ. Macrophage Polarization. Annu Rev Physiol. 2017;79:541-66.

3. Seyedizade SS, Afshari K, Bayat S, Rahmani F, Momtaz S, Rezaei N, et al. Current Status of M1 and M2 Macrophages Pathway as Drug Targets for Inflammatory Bowel Disease. Arch Immunol Ther Exp (Warsz). 2020;68(2):10.

4. Lob S, Konigsrainer A, Rammensee HG, Opelz G, Terness P. Inhibitors of indoleamine-2,3-dioxygenase for cancer therapy: can we see the wood for the trees? Nat Rev Cancer. 2009;9(6):445-52.

5. Zou W. Immunosuppressive networks in the tumour environment and their therapeutic relevance. Nat Rev Cancer. 2005;5(4):263-74.

6. Prendergast GC, Malachowski WJ, Mondal A, Scherle P, Muller AJ. Indoleamine 2,3-Dioxygenase and Its Therapeutic Inhibition in Cancer. Int Rev Cell Mol Biol. 2018;336:175-203.

7. Munn DH, Zhou M, Attwood JT, Bondarev I, Conway SJ, Marshall B, et al. Prevention of allogeneic fetal rejection by tryptophan catabolism. Science. 1998;281(5380):1191-3.

8. Frumento G, Rotondo R, Tonetti M, Damonte G, Benatti U, Ferrara GB. Tryptophan-derived catabolites are responsible for inhibition of $\mathrm{T}$ and natural killer cell proliferation induced by indoleamine 2,3dioxygenase. J Exp Med. 2002;196(4):459-68.

9. Pfefferkorn ER. Interferon gamma blocks the growth of Toxoplasma gondii in human fibroblasts by inducing the host cells to degrade tryptophan. Proc Natl Acad Sci U S A. 1984;81(3):908-12.

10. Fox JM, Sage LK, Poore S, Johnson S, Tompkins SM, Tripp RA. Drug analog inhibition of indoleamine 2,3-dioxygenase (IDO) activity modifies pattern recognition receptor expression and proinflammatory cytokine responses early during influenza virus infection. J Leukocyte Biol. 2014;96(3):447-52.

11. Le Naour J, Galluzzi L, Zitvogel L, Kroemer G, Vacchelli E. Trial watch: IDO inhibitors in cancer therapy. Oncoimmunology. 2020;9(1):1777625.

12. Yue EW, Sparks R, Polam P, Modi D, Douty B, Wayland B, et al. INCB24360 (Epacadostat), a Highly Potent and Selective Indoleamine-2,3-dioxygenase 1 (ID01) Inhibitor for Immuno-oncology. ACS Med Chem Lett. 2017;8(5):486-91.

13. Long GV, Dummer R, Hamid O, Gajewski TF, Caglevic C, Dalle S, et al. Epacadostat plus pembrolizumab versus placebo plus pembrolizumab in patients with unresectable or metastatic melanoma (ECHO-301/KEYNOTE-252): a phase 3, randomised, double-blind study. Lancet Oncol. 2019;20(8):1083-97.

14. Ouchi Y, Yoshikawa E, Sekine Y, Futatsubashi M, Kanno T, Ogusu T, et al. Microglial activation and dopamine terminal loss in early Parkinson's disease. Ann Neurol. 2005;57(2):168-75.

15. Li R, Huang YG, Fang D, Le WD. (-)-Epigallocatechin gallate inhibits lipopolysaccharide-induced microglial activation and protects against inflammation-mediated dopaminergic neuronal injury. $\mathrm{J}$ Neurosci Res. 2004;78(5):723-31.

16. Liu YC, Zou XB, Chai YF, Yao YM. Macrophage polarization in inflammatory diseases. Int J Biol Sci. 2014;10(5):520-9. 
17. Gao HM, Liu B, Zhang W, Hong JS. Critical role of microglial NADPH oxidase-derived free radicals in the in vitro MPTP model of Parkinson's disease. FASEB J. 2003;17(13):1954-6.

18. Martinez FO, Gordon S. The M1 and M2 paradigm of macrophage activation: time for reassessment. F1000Prime Rep. 2014;6:13.

19. Ma L, Hu B, Liu Y, Vermilyea SC, Liu H, Gao L, et al. Human embryonic stem cell-derived GABA neurons correct locomotion deficits in quinolinic acid-lesioned mice. Cell Stem Cell. 2012;10(4):45564.

20. McWhorter FY, Wang T, Nguyen P, Chung T, Liu WF. Modulation of macrophage phenotype by cell shape. Proc Natl Acad Sci U S A. 2013;110(43):17253-8.

21. Croitoru-Lamoury J, Lamoury FM, Caristo M, Suzuki K, Walker D, Takikawa O, et al. Interferon-gamma regulates the proliferation and differentiation of mesenchymal stem cells via activation of indoleamine 2,3 dioxygenase (IDO). PLoS One. 2011;6(2):e14698.

22. Konan KV, Taylor MW. Importance of the two interferon-stimulated response element (ISRE) sequences in the regulation of the human indoleamine 2,3-dioxygenase gene. J Biol Chem. 1996;271(32):19140-5.

23. Folkman J, Moscona A. Role of cell shape in growth control. Nature. 1978;273(5661):345-9.

24. Horsthemke M, Bachg AC, Groll K, Moyzio S, Muther B, Hemkemeyer SA, et al. Multiple roles of filopodial dynamics in particle capture and phagocytosis and phenotypes of Cdc42 and Myo10 deletion. J Biol Chem. 2017;292(17):7258-73.

25. Racoosin EL, Swanson JA. M-CSF-induced macropinocytosis increases solute endocytosis but not receptor-mediated endocytosis in mouse macrophages. J Cell Sci. 1992;102 (Pt 4):867-80.

26. Swanson JA, Watts C. Macropinocytosis. Trends Cell Biol. 1995;5(11):424-8.

27. Canton J, Schlam D, Breuer C, Gutschow M, Glogauer M, Grinstein S. Calcium-sensing receptors signal constitutive macropinocytosis and facilitate the uptake of NOD2 ligands in macrophages. Nat Commun. 2016;7:11284.

28. Qie Y, Yuan H, von Roemeling CA, Chen Y, Liu X, Shih KD, et al. Surface modification of nanoparticles enables selective evasion of phagocytic clearance by distinct macrophage phenotypes. Sci Rep. 2016;6:26269.

29. Akilbekova D, Philiph R, Graham A, Bratlie KM. Macrophage reprogramming: influence of latex beads with various functional groups on macrophage phenotype and phagocytic uptake in vitro. J Biomed Mater Res A. 2015;103(1):262-8.

30. Hind LE, Lurier EB, Dembo M, Spiller KL, Hammer DA. Effect of M1-M2 Polarization on the Motility and Traction Stresses of Primary Human Macrophages. Cell Mol Bioeng. 2016;9(3):455-65.

31. Cui K, Ardell CL, Podolnikova NP, Yakubenko VP. Distinct Migratory Properties of M1, M2, and Resident Macrophages Are Regulated by alphaDbeta2 and alphaMbeta2 Integrin-Mediated Adhesion. Front Immunol. 2018;9:2650. 
32. Swanson KV, Deng M, Ting JP. The NLRP3 inflammasome: molecular activation and regulation to therapeutics. Nat Rev Immunol. 2019;19(8):477-89.

33. Zhang J, Liu X, Wan C, Liu Y, Wang Y, Meng C, et al. NLRP3 inflammasome mediates M1 macrophage polarization and IL-1beta production in inflammatory root resorption. J Clin Periodontol. 2020;47(4):451-60.

34. Zhang WY, Guo YJ, Han WX, Yang MQ, Wen LP, Wang KY, et al. Curcumin relieves depressive-like behaviors via inhibition of the NLRP3 inflammasome and kynurenine pathway in rats suffering from chronic unpredictable mild stress. Int Immunopharmacol. 2019;67:138-44.

35. Saxton RA, Sabatini DM. mTOR Signaling in Growth, Metabolism, and Disease. Cell. 2017;169(2):361-71.

36. Metz R, Rust S, Duhadaway JB, Mautino MR, Munn DH, Vahanian NN, et al. IDO inhibits a tryptophan sufficiency signal that stimulates mTOR: A novel IDO effector pathway targeted by D-1-methyltryptophan. Oncoimmunology. 2012;1(9):1460-8.

37. Labadie BW, Bao R, Luke JJ. Reimagining IDO Pathway Inhibition in Cancer Immunotherapy via Downstream Focus on the Tryptophan-Kynurenine-Aryl Hydrocarbon Axis. Clin Cancer Res. 2019;25(5):1462-71.

38. Moon JS, Hisata S, Park MA, DeNicola GM, Ryter SW, Nakahira K, et al. mTORC1-Induced HK1Dependent Glycolysis Regulates NLRP3 Inflammasome Activation. Cell Rep. 2015;12(1):102-15.

39. Zhang S, Zong Y, Ren Z, Hu J, Wu X, Xiao H, et al. Regulation of indoleamine 2, 3-dioxygenase in hippocampal microglia by NLRP3 inflammasome in lipopolysaccharide-induced depressive-like behaviors. Eur J Neurosci. 2020.

40. Coll RC, Hill JR, Day CJ, Zamoshnikova A, Boucher D, Massey NL, et al. MCC950 directly targets the NLRP3 ATP-hydrolysis motif for inflammasome inhibition. Nat Chem Biol. 2019;15(6):556-9.

41. He H, Jiang $\mathrm{H}$, Chen $\mathrm{Y}$, Ye J, Wang A, Wang $\mathrm{C}$, et al. Oridonin is a covalent NLRP3 inhibitor with strong anti-inflammasome activity. Nat Commun. 2018;9(1):2550.

42. Prendergast GC, Malachowski WP, DuHadaway JB, Muller AJ. Discovery of IDO1 Inhibitors: From Bench to Bedside. Cancer Res. 2017;77(24):6795-811.

43. Pallotta MT, Orabona C, Volpi C, Vacca C, Belladonna ML, Bianchi R, et al. Indoleamine 2,3dioxygenase is a signaling protein in long-term tolerance by dendritic cells. Nat Immunol. 2011;12(9):870-8.

44. Sims JE, Smith DE. The IL-1 family: regulators of immunity. Nat Rev Immunol. 2010;10(2):89-102.

45. Fukuwatari T, Shibata K. Nutritional aspect of tryptophan metabolism. Int J Tryptophan Res. 2013;6(Suppl 1):3-8.

46. Revathikumar P, Bergqvist F, Gopalakrishnan S, Korotkova M, Jakobsson PJ, Lampa J, et al. Immunomodulatory effects of nicotine on interleukin 1 beta activated human astrocytes and the role of cyclooxygenase 2 in the underlying mechanism. J Neuroinflammation. 2016;13(1):256. 
47. Lipszyc PS, Cremaschi GA, Zorrilla-Zubilete M, Bertolino ML, Capani F, Genaro AM, et al. Niacin Modulates Pro-inflammatory Cytokine Secretion. A Potential Mechanism Involved in its Antiatherosclerotic Effect. Open Cardiovasc Med J. 2013;7:90-8.

48. Montserrat-de la Paz S, Naranjo MC, Lopez S, Abia R, Muriana FJG, Bermudez B. Niacin and its metabolites as master regulators of macrophage activation. J Nutr Biochem. 2017;39:40-7.

49. Jeon SA, Lee E, Hwang I, Han B, Park S, Son S, et al. NLRP3 Inflammasome Contributes to Lipopolysaccharide-induced Depressive-Like Behaviors via Indoleamine 2,3-dioxygenase Induction. Int J Neuropsychopharmacol. 2017;20(11):896-906.

50. Companies Scaling Back ID01 Inhibitor Trials. Cancer Discov. 2018;8(7):OF5.

\section{Figures}



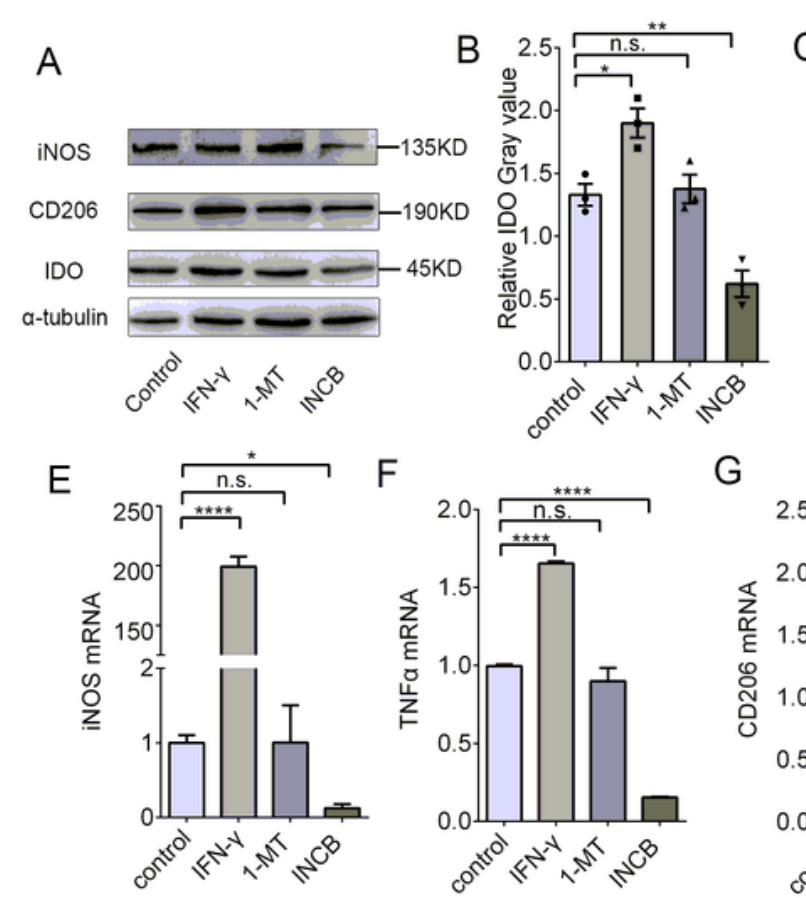

I
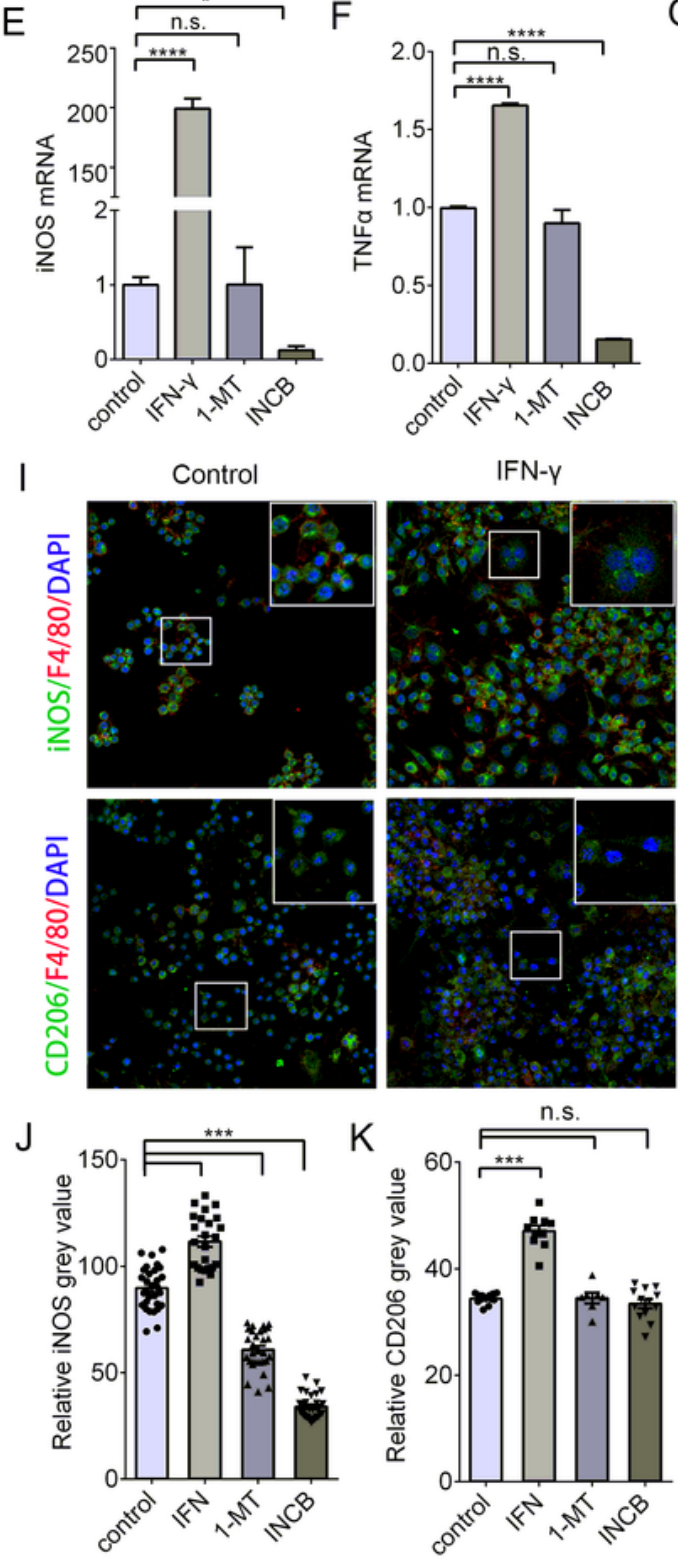

G

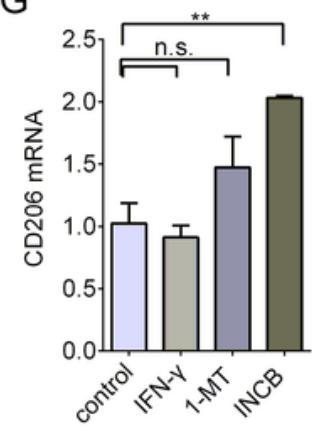

1-MT
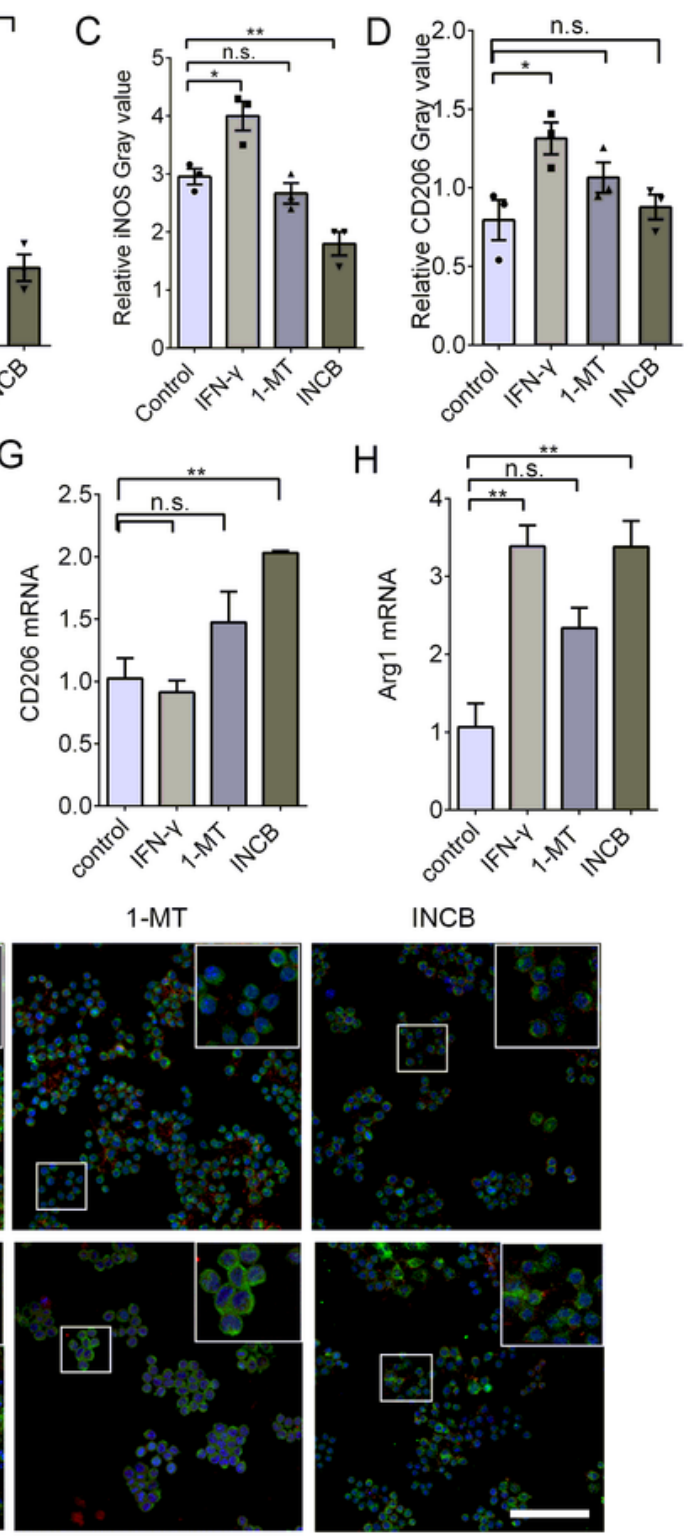

Fig.1

\section{Figure 1}

Inhibition of IDO-1 with 1-MT and INCB-24360 decreased iNOS and TNF-a A-D. IDO-1, iNOS, and CD206 expression in RAW264.7 treated with IFN- $\gamma, 1-M T$ and INCB for 24h (A). The relative intensity of ID0-1 (B), iNOS (C), and C D206 (D) in RAW264.7 measured by ImageJ. E-H. The transcription levels of iNOS (E), TNFa (F), CD206 (J) and Arg1 (H) in RAW264.7 treated with IFN-y, 1-MT and INCB for 24h. I-k. The immunostaining images of iNOS and CD206 in RAW264.7 treated by IFN- - , 1-MT and INCB for 24h. The 
relative intensity of iNOS (J) or CD206 (K) in RAW264.7 after treatment with IFN- $\gamma, 1-M T$ and INCB, which measured by ImageJ. $n \geq 10$. Scale bars, $100 \mu$ m. One-way ANOVA; all data are expressed as the mean \pm SEM. *, $\mathrm{P}<0.05$, **, $\mathrm{P}<0.01 ;$ ns, no statistical difference.
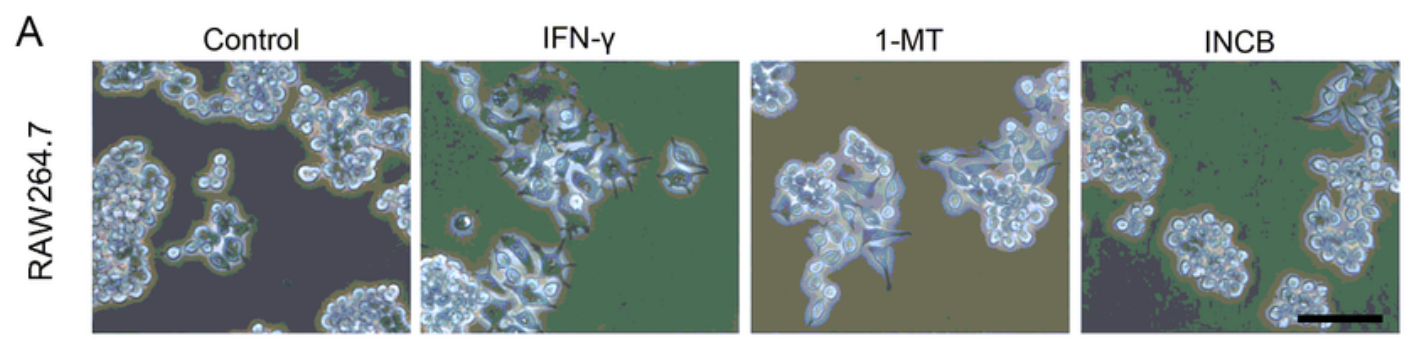

B

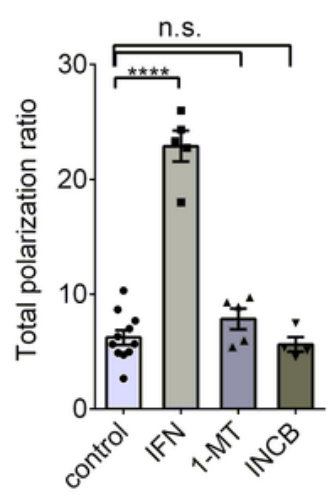

C

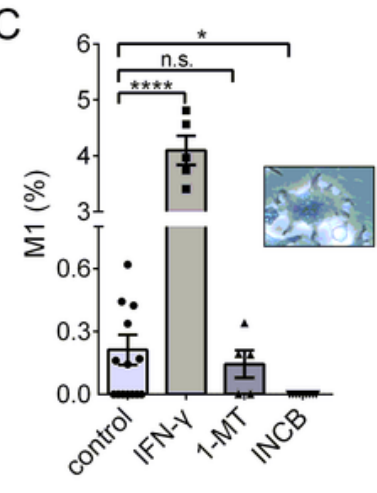

D

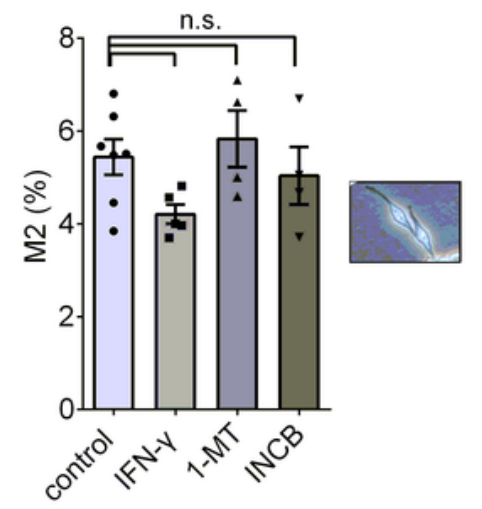

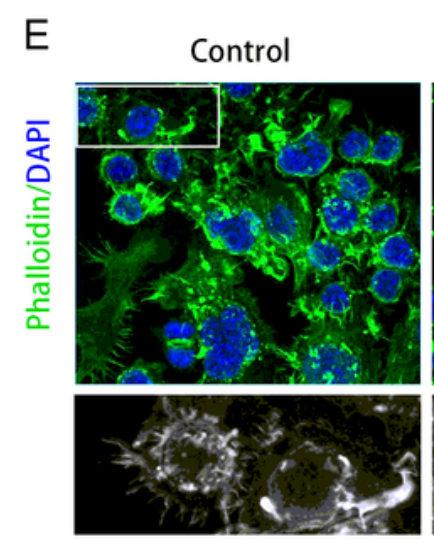

$\mathrm{F}$

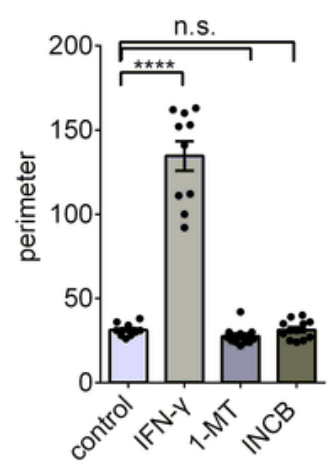

IFN- $\gamma$
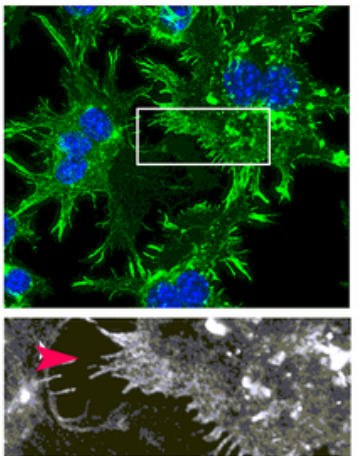

G

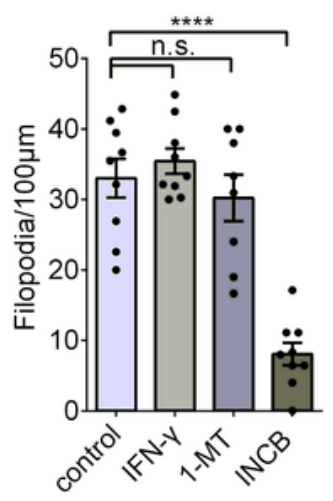

1-MT
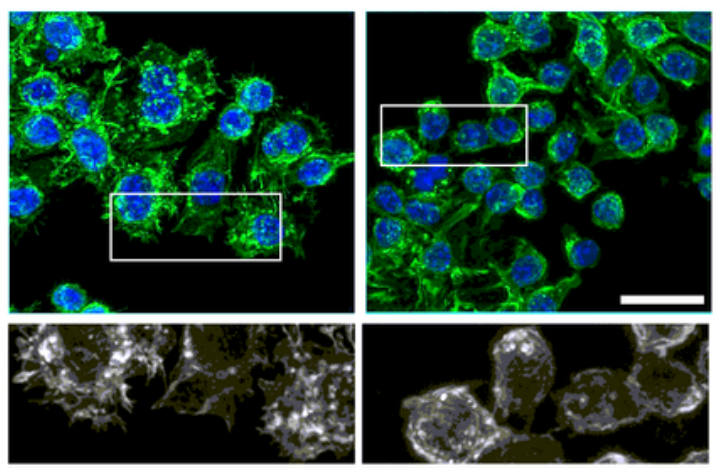

Fig.2

Figure 2

1-MT and INCB-24360 treatment reduced M1-like not M2-like RAW264.7 A-D. The typical morphology of RAW264.7 treated with IFN-ץ, 1-MT and INCB for 24h. More round and small RAW264.7 were observed in 
1-MT and INCB groups compared to IFN- $\gamma$ and control group (A). The percentage of the total polarized (B), M1-type-cells (ramified) (C), and M2-type-cells (slender) (D) in the control, IFN- $\gamma, 1-M T$ and INCB groups. N $\geq 5$. Scale bars, $100 \mu \mathrm{m}$. E-G. The phalloidin Alexa-488 staining of RAW264.7 treated with IFN-ץ, 1-MT and INCB for 24h. The cellular perimeters in the control, IFN-y, 1-MT and INCB groups (F). The density of filopodia on the membrane of RAW264.7 in the control, IFN-y, 1-MT and INCB groups (G). $n \geq 10$. Scale bars, $30 \mu \mathrm{m}$. One-way ANOVA; all data are expressed as the mean $\pm \mathrm{SEM} .{ }^{*}, \mathrm{P}<0.05, * \star, P<0.01$; ns, no statistical difference.
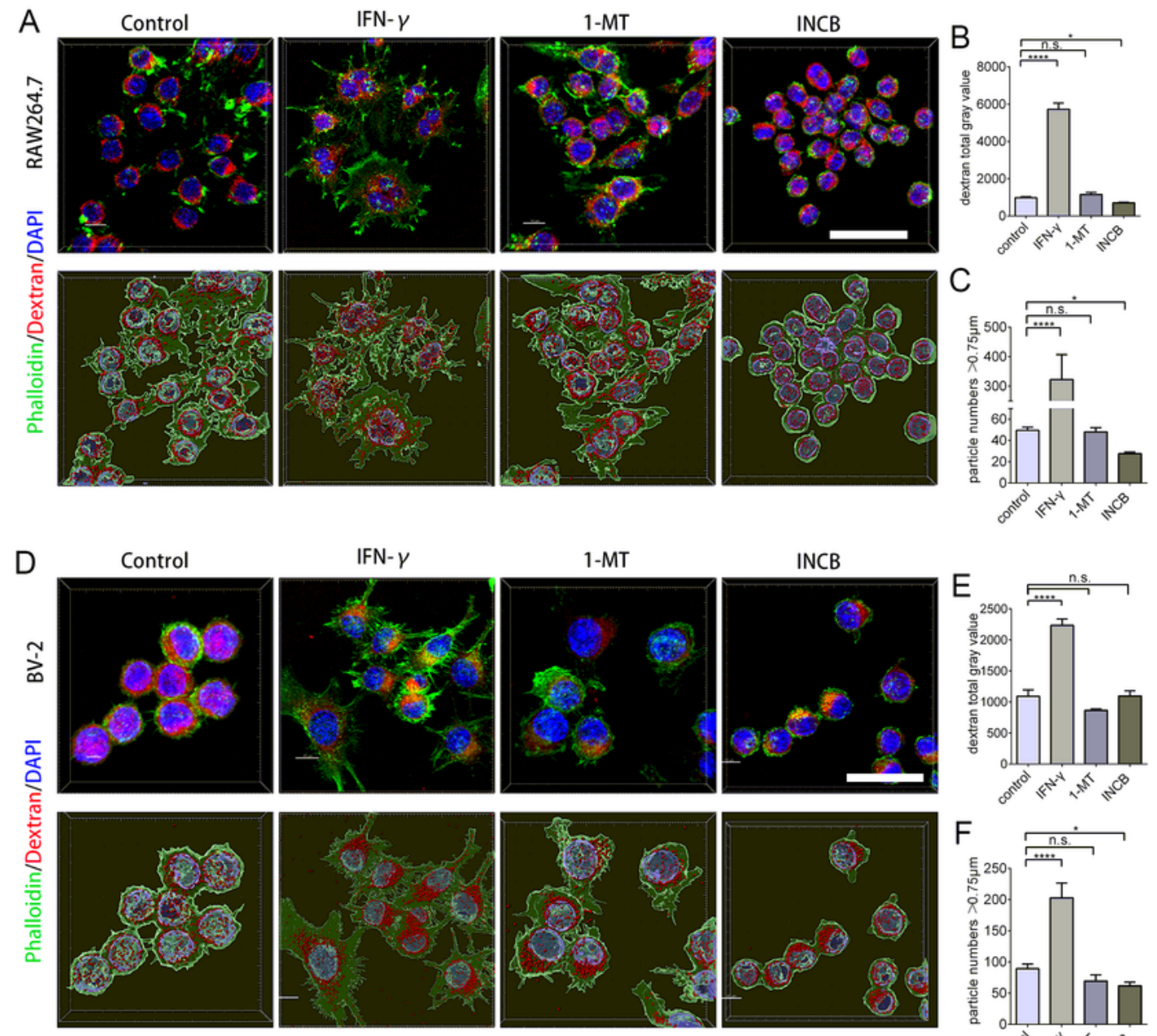

G

Control
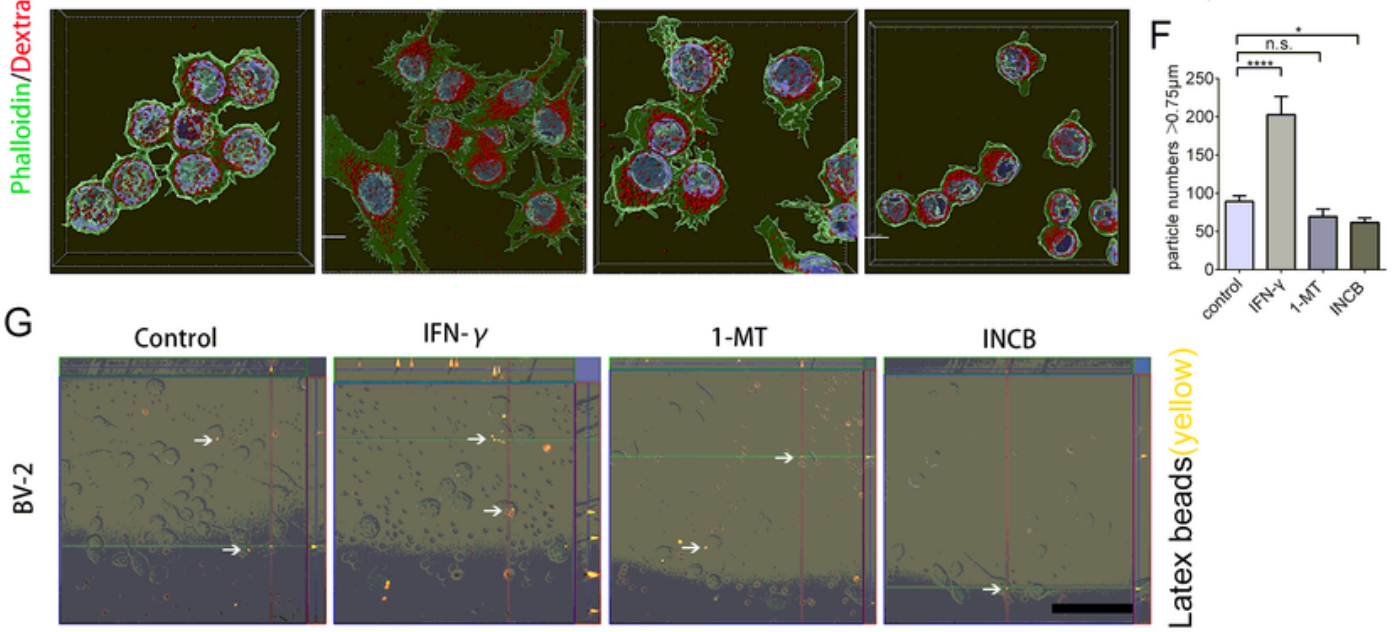

IFN- $\gamma$

1-MT

INCB
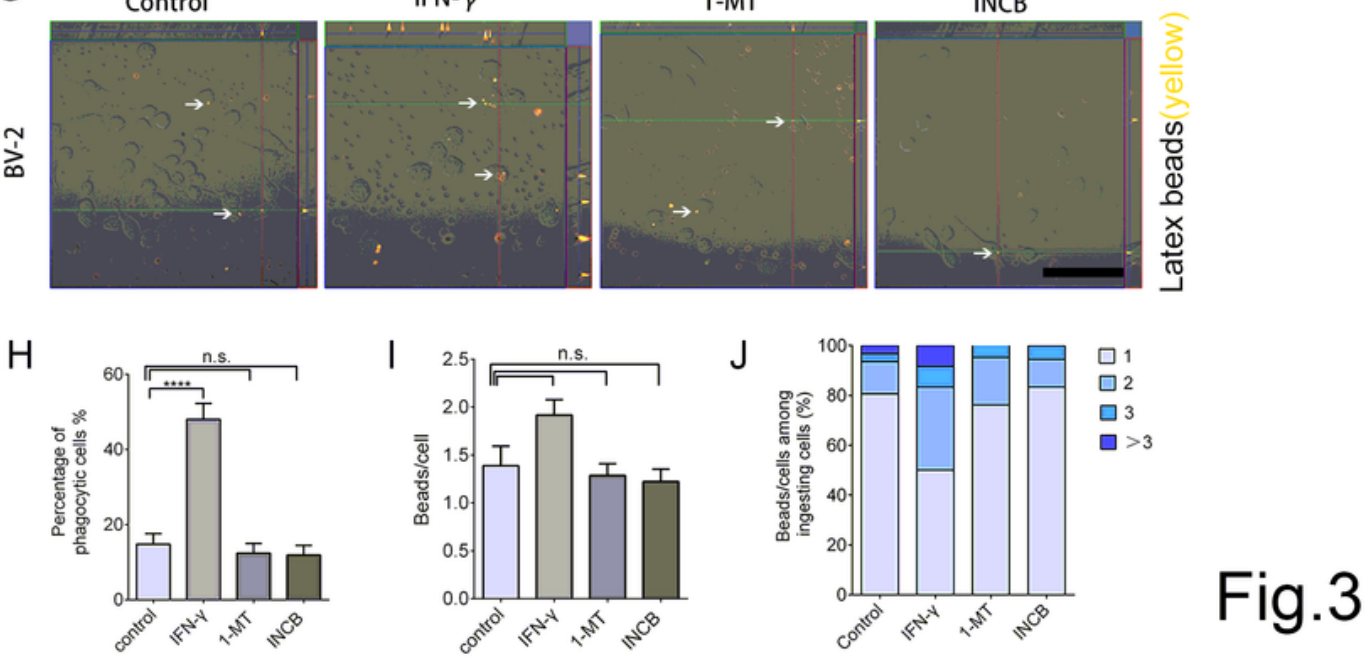


\section{Figure 3}

INCB-24360 treatment reduced DTR-Dextran uptake in RAW264.7 and BV-2 A, D. The typical images of engulfed red fluorescent Dextran in RAW264.7 or BV-2 cells after administration of dextran for 1 hour in control, IFN- $y, 1-M T$ and INCB groups. The phagocytic vesicles that are larger than $0.75 \mu \mathrm{m}$ were visualized by the spot function of IMARIS software 9.5. Scale bars, $40 \mu \mathrm{m}$. B, E. Quantification of the engulfed Dextran particles in RAW264.7 (B) and BV-2(E) cells using ImageJ. Single-cell area X Dextran average gray-scale was used as statistical data, $n \geq 15$. C, F. Spots numbers which represent particles that are larger than $0.75 \mu \mathrm{m}$ in control, IFN- $\gamma, 1-M T$ and INCB groups of RAW264.7(C) or BV2 (F) cells. $n \geq$ 15. G-J. Phagocytic latex beads in BV-2 treated with IFN-y, 1-MT and INCB for 24h (G). The phagocytic ratio $(H)$, the number of beads of each cell $(\mathrm{I})$, the number of cell of one, two, three, or more beads $(\mathrm{J})$. Scale bars, $100 \mu \mathrm{m}$. One-way ANOVA; all data are expressed as the mean $\pm \mathrm{SEM}$. ${ }^{*}, \mathrm{P}<0.05,{ }^{\star *}, \mathrm{P}<0.01$; $\mathrm{ns}$, no statistical difference. 

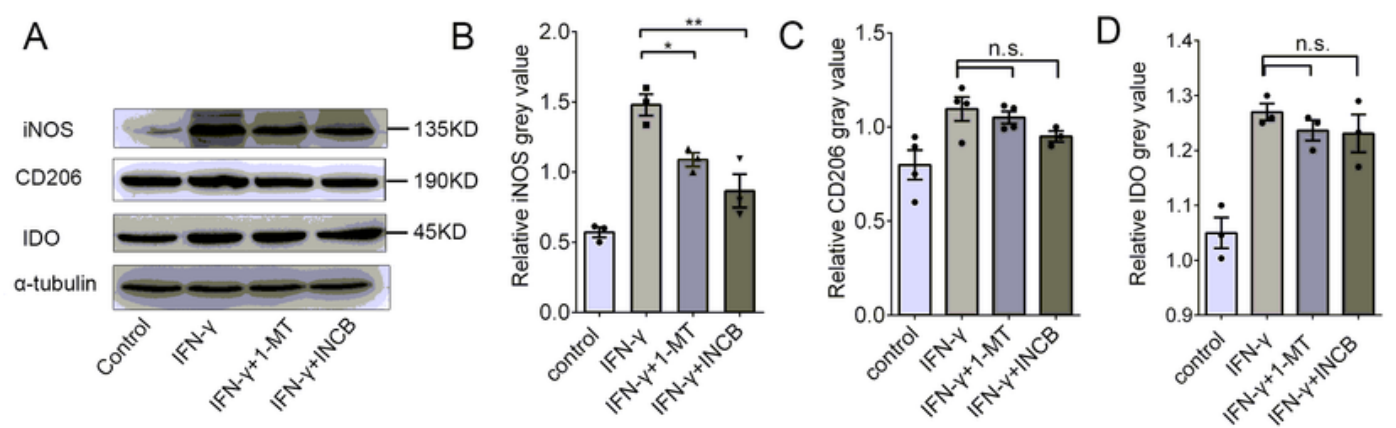

E

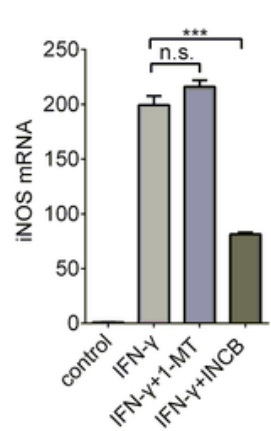

।
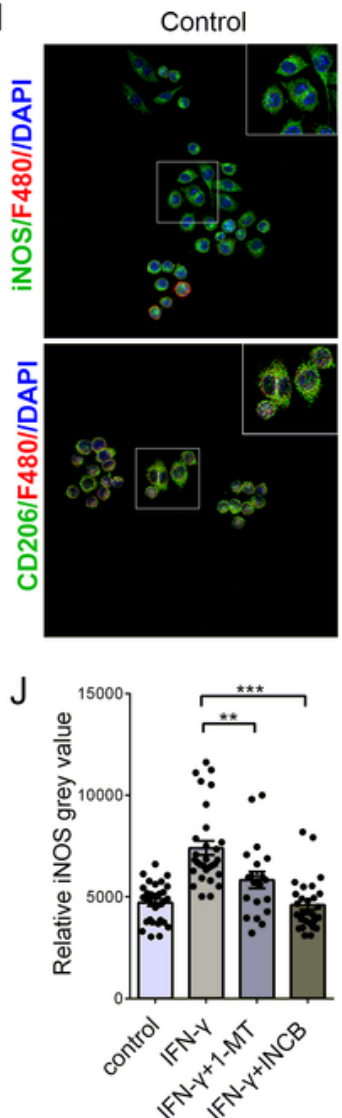
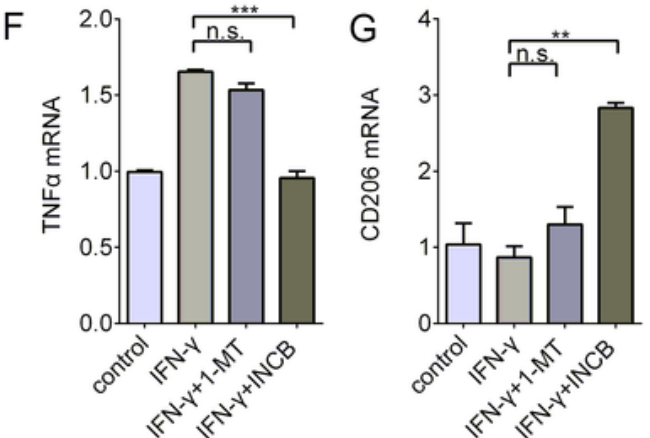

$\leqslant 0$

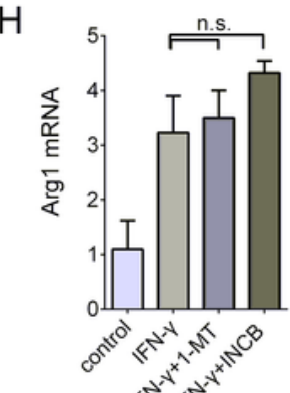

IFN- $\mathrm{y}+\mathrm{INCB}$
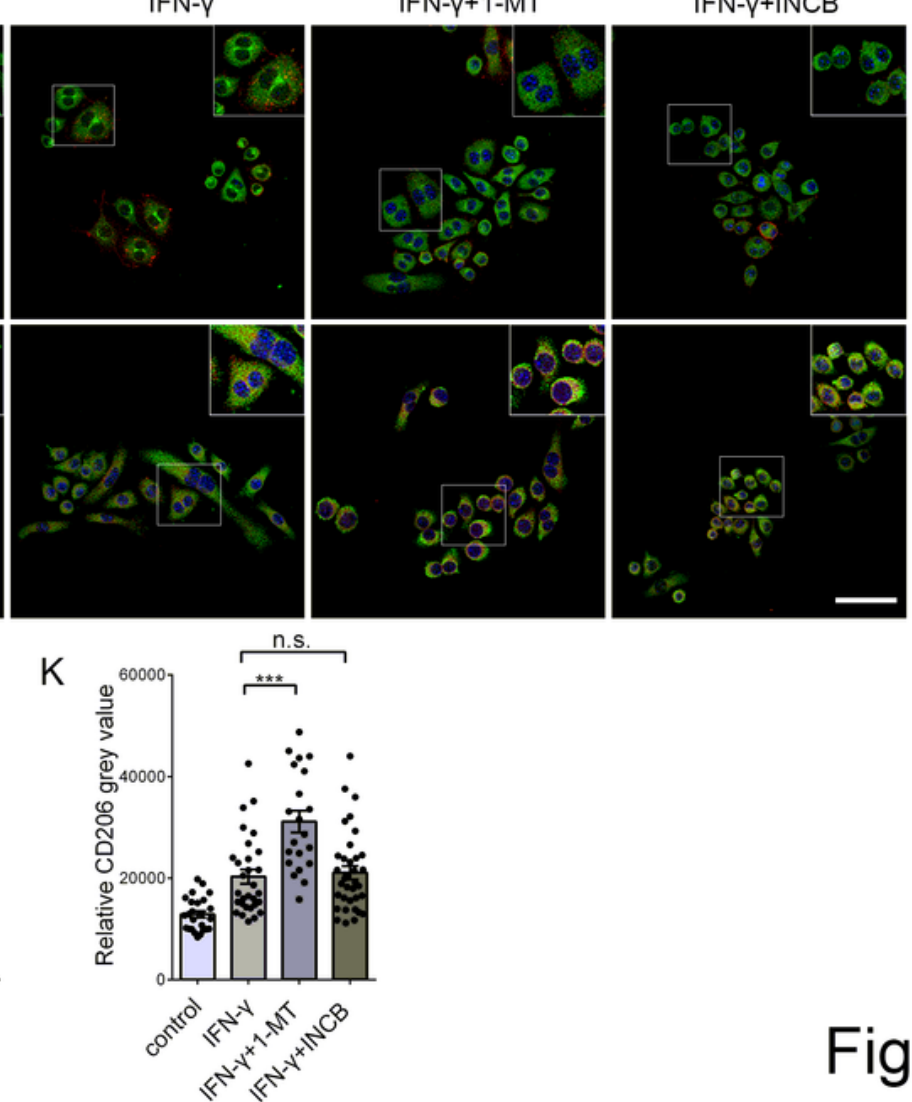

Fig.4

\section{Figure 4}

INCB24360 and 1-MT suppresses IFN-y induced iNOS and TNFa increase in RAW264.7 A-D. iNOS, CD206 and ID0-1 expression in RAW264.7 treated with IFN- $y$, IFN- $-1+1-M T$ and IFN- $\gamma+$ INCB for $24 \mathrm{~h}$ (12 hours with IFN- $\gamma$, following another 12 hours' supplement with 1-MT or INCB, respectively), the relative intensity of iNOS (B), CD206 (C), and IDO-1 (D) in RAW264.7 measured by ImageJ. E-H. The transcription levels of iNOS (E), TNFa (F), CD206 (G) and Arg1 (H) in RAW264.7 treated with IFN- - , IFN- - +1-MT and IFN- $-\gamma+$ INCB 
for 24h. I-K. The immunostaining images of iNOS and CD206 in RAW264.7 treated by IFN- $\mathrm{y}$, IFN- $\mathrm{y}+1-\mathrm{MT}$ and IFN- $\gamma+$ INCB for 24h. The quantification of iNOS (J) or CD206 (K) intensity in RAW264.7 measured by

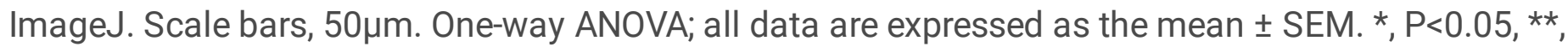
$\mathrm{P}<0.01$; ns, no statistical difference.

A
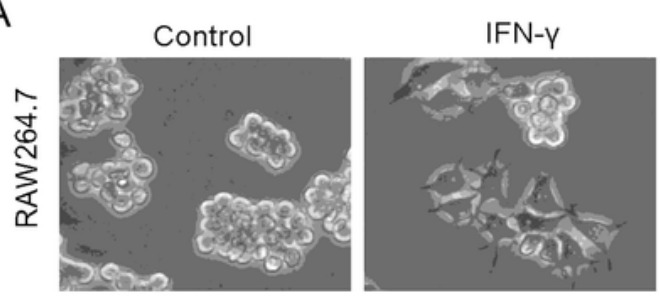

IFN- $\mathrm{Y}+1-\mathrm{MT}$

IFN- $\gamma+$ INCB
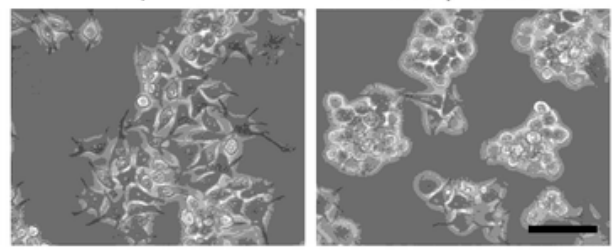

B
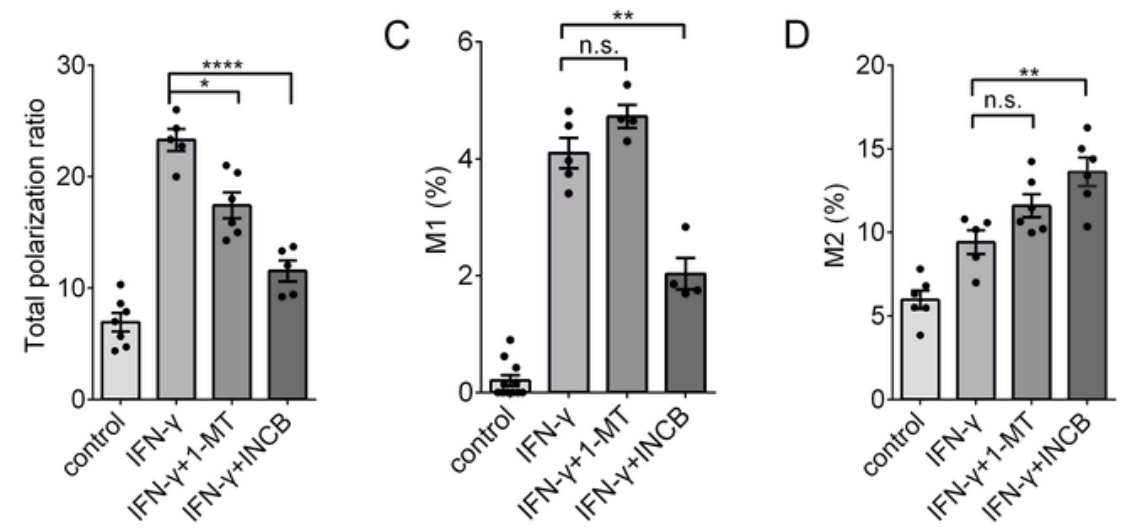

$\mathrm{E}$
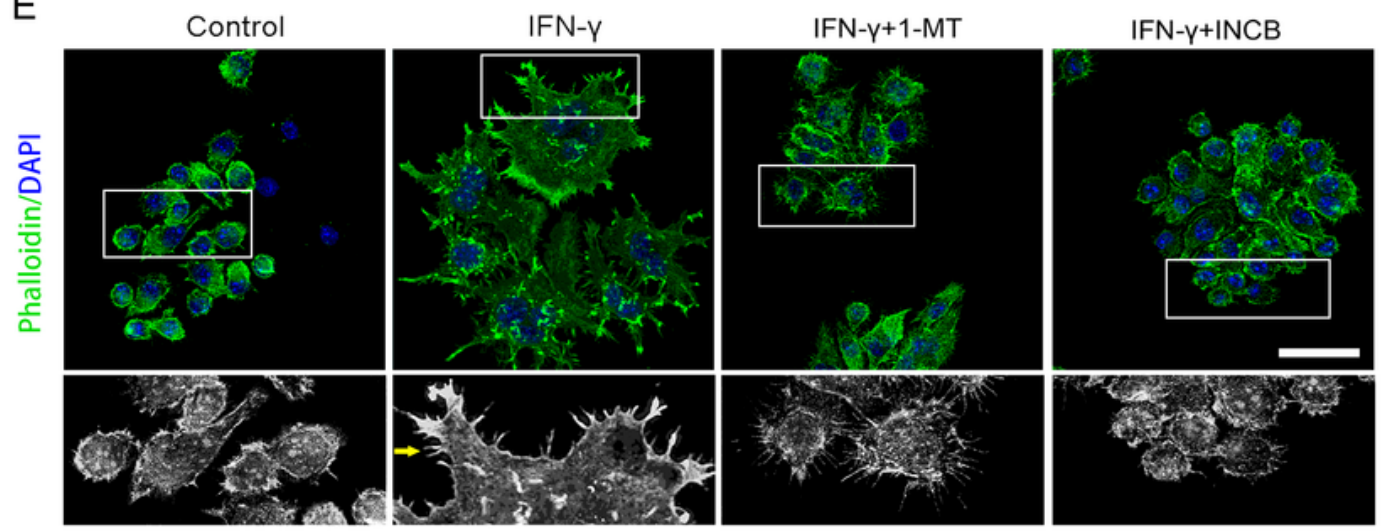

$\mathrm{F}$

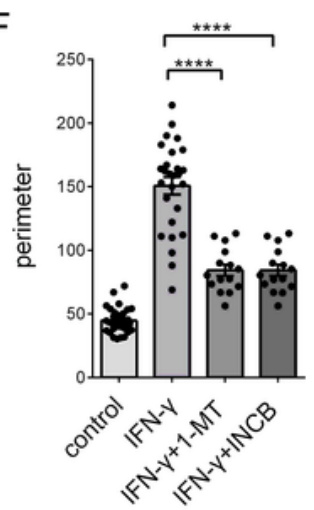

G

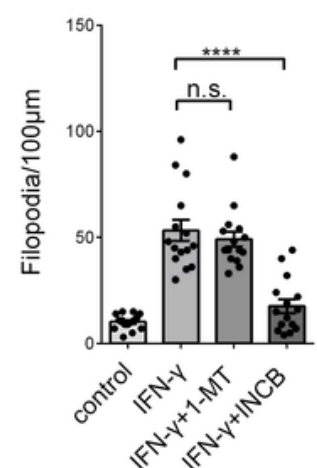

Fig.5

\section{Figure 5}


INCB-24360 treatment reduced M1-like macrophage in RAW264.7 induced by IFN-ץ A-D. The typical morphology of RAW264.7 after treating with IFN- - , IFN- $\gamma+1-M T$ and IFN- $\gamma+$ INCB. More round and small RAW264.7 were observed in IFN- $\gamma+1-M T$ and IFN- $\gamma+$ INCB24360 groups compared to IFN- $\gamma$ group (A). The percentage of the polarized (B), M1-like macrophage (ramified)(C), and M2-like macrophage (slender) (D) in the control, IFN- $\gamma$, IFN- $\gamma+1-M T$ and IFN- $\gamma+$ INCB groups. $N \geq 5$. Scale bars, $60 \mu \mathrm{m}$. E-G. The phalloidin Alexa-488 staining of RAW264.7 treated by IFN- $\gamma$, IFN- $-1+1-M T$ and IFN- $\gamma+$ INCB for $24 \mathrm{~h}$. The cellular perimeters in the control, IFN- $\gamma$, IFN- $\gamma+1-M T$ and IFN- $\gamma+$ INCB groups (F). The density of filopodia of RAW264.7 in the control, IFN- - , IFN- $\gamma+1-M T$ and IFN- $\gamma+$ INCB groups. (G). One-way ANOVA; all data are expressed as the mean \pm SEM. * $, \mathrm{P}<0.05, * \star, P<0.01$; ns, no statistical difference. Scale bars, $40 \mu \mathrm{m}$. 
A
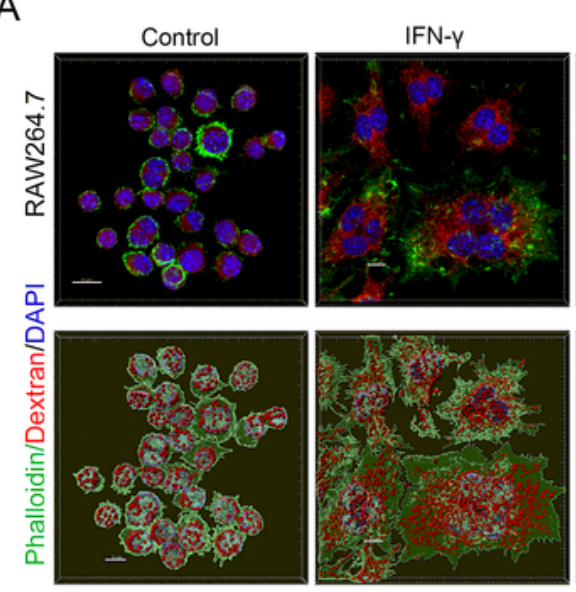

D
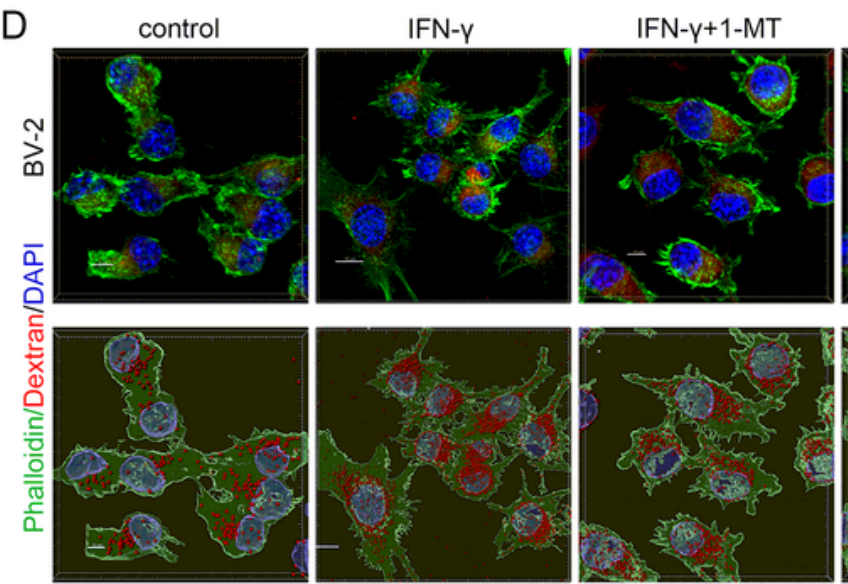

EN

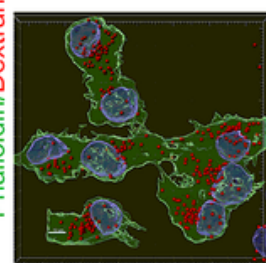

G

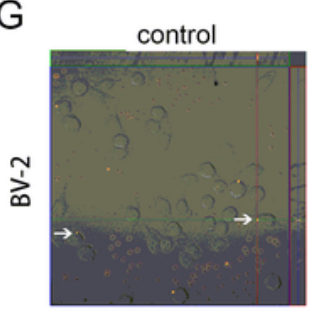

$\mathrm{H}$
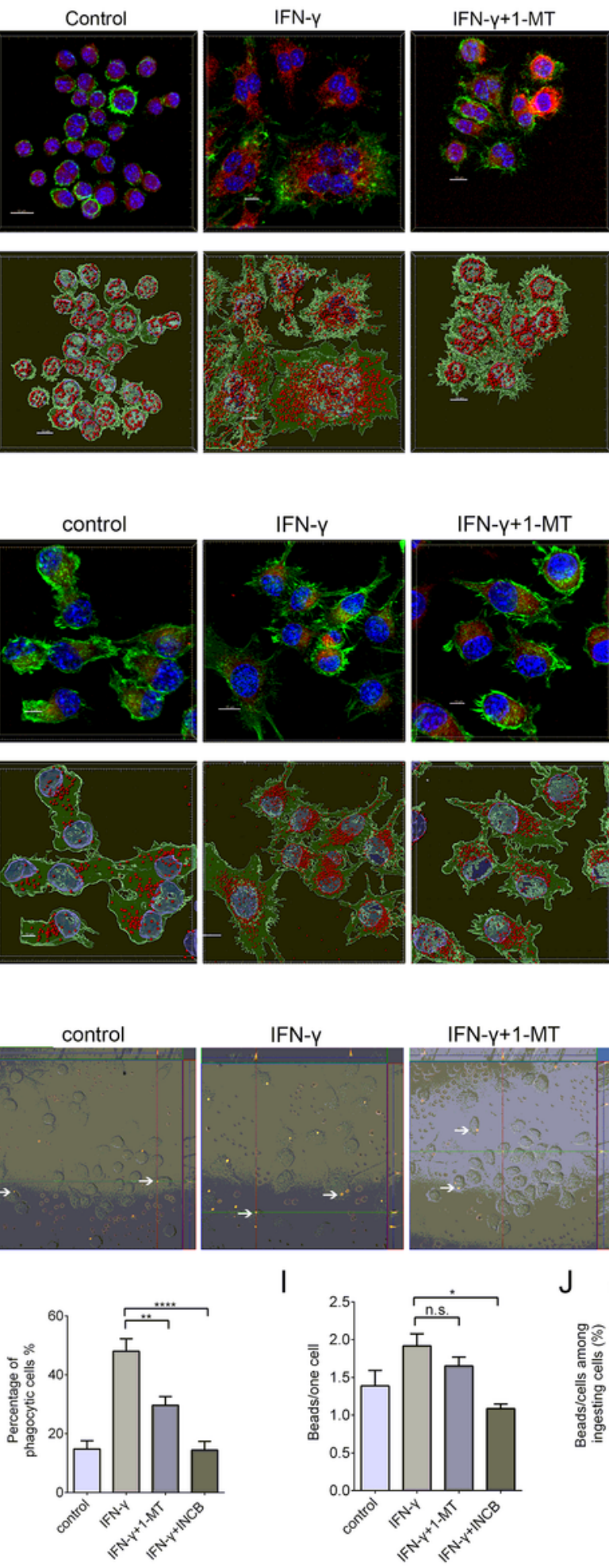

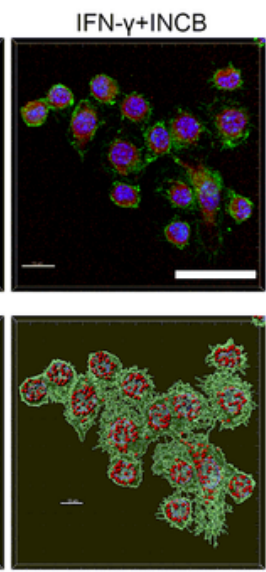

$B$
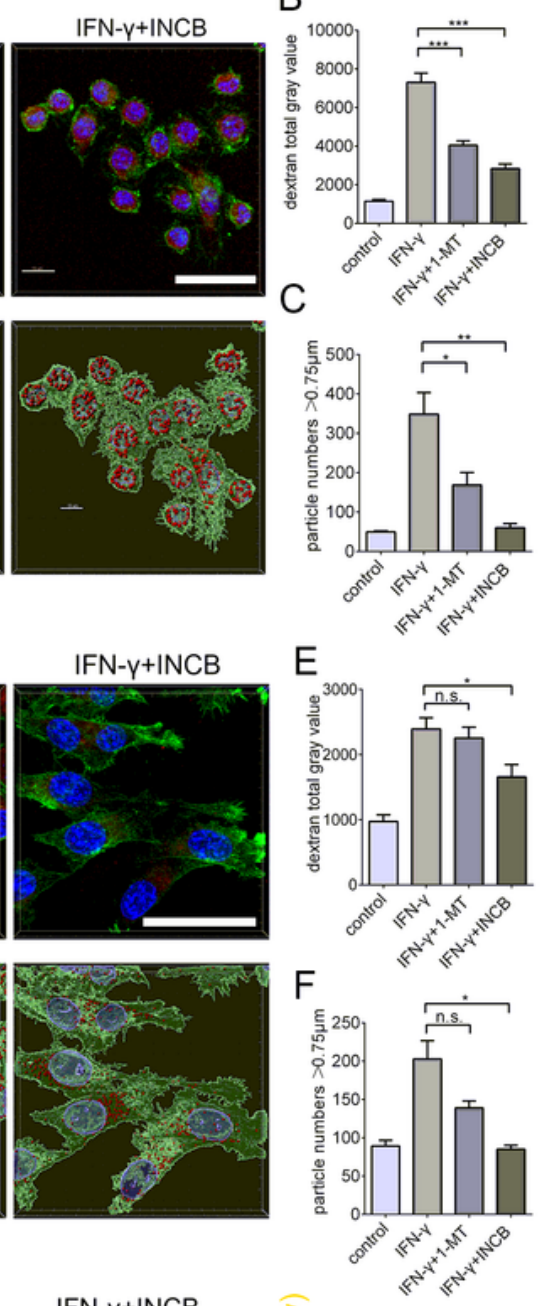

IFN- $\gamma+$ INCB
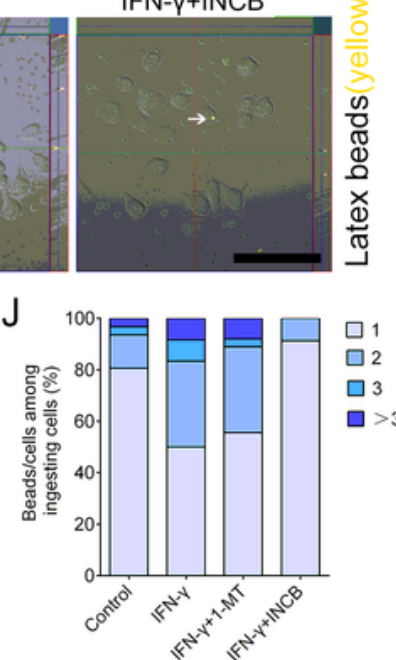

Fig.6

\section{Figure 6}

1-MT and INCB-24360 reduced DTR-Dextran uptake in RAW264.7 and BV-2 induced by IFN-ץ A, D. The typical images of engulfed DTR-Dextran in IFN- $\gamma$ induced RAW264.7 or BV-2 cells after administration of dextran for 1 hour in the control, IFN- $\gamma$, IFN- $\gamma+1-M T$ and IFN- $\gamma+$ INCB groups. The phagocytic particles that

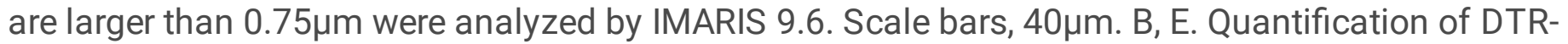
Dextran particles in RAW264.7 (B) and BV-2 (E). Single-cell area X Dextran average gray-scale was used 
as statistical data, $n \geq 15$. C, F. DTR-dextran particles that are larger than $0.75 \mu \mathrm{m}$ in the control, IFN- $y$, IFN- $\gamma+1-M T$ and IFN- $\gamma+$ INCB groups of IFN- $\gamma$ induced RAW264.7(C) or BV2(F) cells. $n \geq 15$. G-J.

Phagocytosis of latex beads in BV-2 treated with IFN- - , IFN- $\gamma+1-M T$ and IFN- $y+$ INCB for $24 \mathrm{~h}(\mathrm{G})$. The phagocytic ratio $(H)$, the number of beads of each cell $(I)$, the number of cell of one, two, three, or more beads $(J)$. Scale bars, $100 \mu \mathrm{m}$. One-way ANOVA; all data are expressed as the mean $\pm \mathrm{SEM}$. *, $\mathrm{P}<0.05$, **, $P<0.01 ;$ ns, no statistical difference.

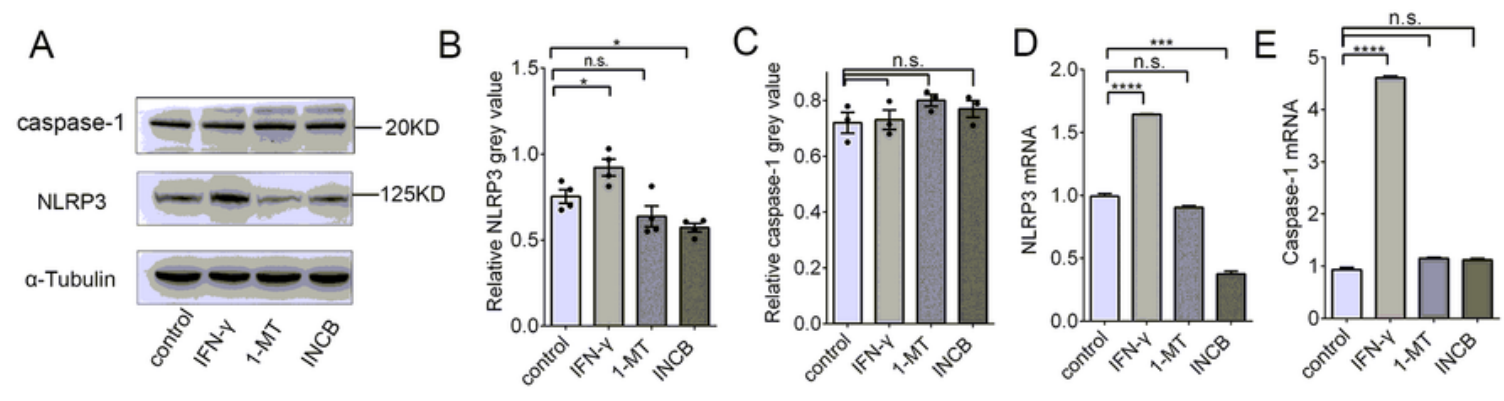

F

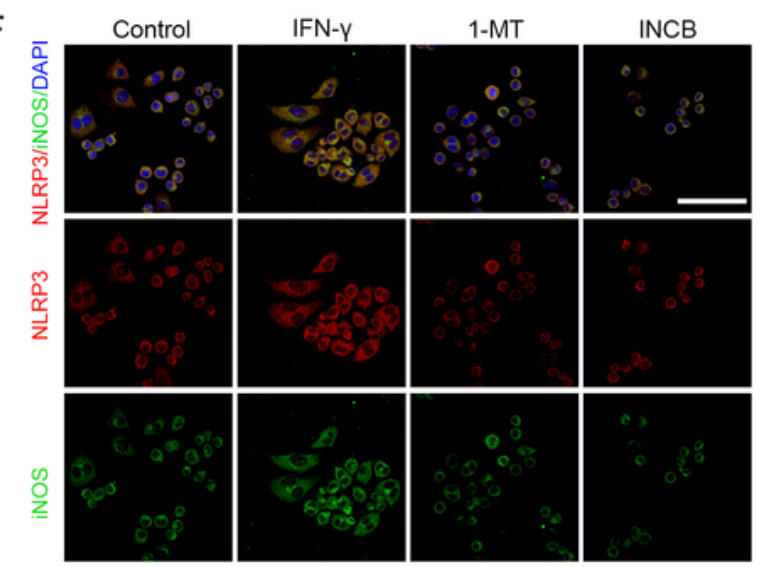

G
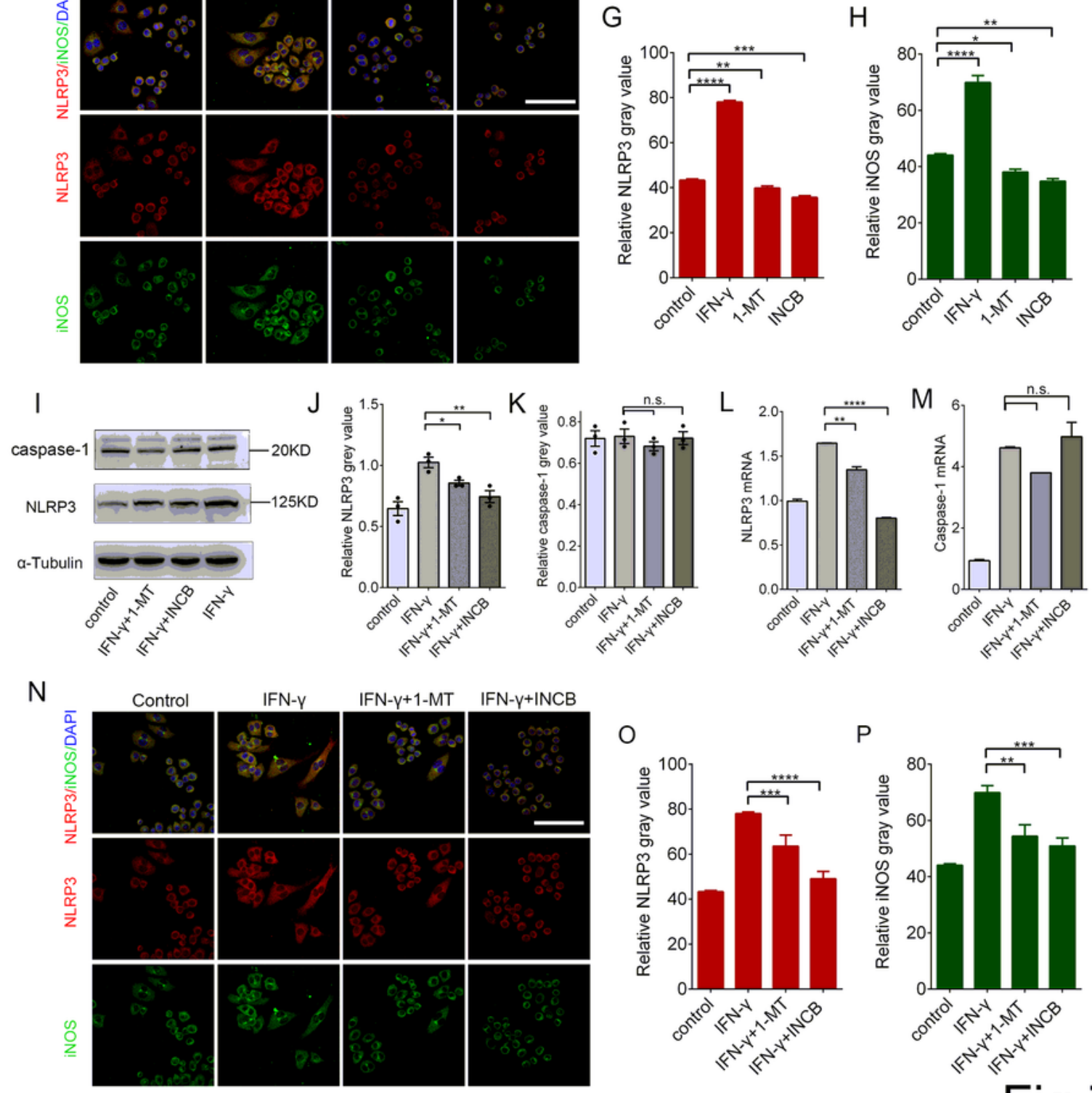

Fig.7

Figure 7 
1-MT and INCB24360 reduced NLRP3 expression and NLRP3 gene transcription in RAW264.7 A-C. The changes of NLRP3 and caspase-1 in RAW264.7 treated with IFN-y, 1-MT and INCB for 24h (A). The relative intensity of NLRP3 (B), caspase-1 (C) in RAW264.7 measured by ImageJ. D, E. The transcription levels of NLRP3 and caspase-1 genes in RAW264.7 treated with IFN- $\gamma, 1-M T$ and INCB for 24h. F-H. The immunostaining results of NLRP3 and iNOS in RAW264.7 treated with IFN- - , 1-MT and INCB for 24h. The

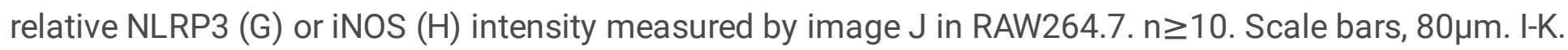
The changes of NLRP3 and caspase-1 in RAW264.7 treated with IFN- $\gamma$, IFN- $-+1-M T$ and IFN- $-\gamma+$ INCB for $24 \mathrm{~h}(\mathrm{I})$. The relative intensity of NLRP3 (J), caspase-1 (K) in RAW264.7 measured by Image J. L, M. The transcription levels of NLRP3 and caspase-1 in RAW264.7 after treated with IFN- $\gamma$, IFN- $\gamma+1-\mathrm{MT}$ and IFN- $\gamma$ + INCB for 24h. N-P. The immunostaining images of NLRP3 and iNOS in RAW264.7 treated with IFN- $\gamma$, IFN$\gamma+1-M T$ and IFN- $\gamma+$ INCB for $24 \mathrm{~h}$. The relative NLRP3 (O) or iNOS (P) intensity measured by ImageJ in RAW264.7. Scale bars are 80 $\mu \mathrm{m}$. One-way ANOVA; all data are expressed as the mean \pm SEM. ${ }^{*}, P<0.05$, **, $\mathrm{P}<0.01 ;$ ns, no statistical difference. Scale bars, $80 \mu \mathrm{m}$. 
A

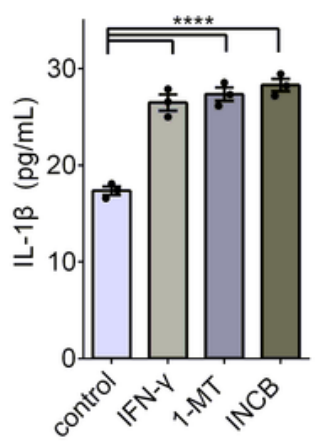

D

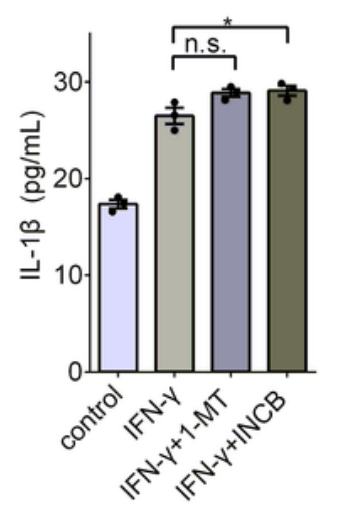

G

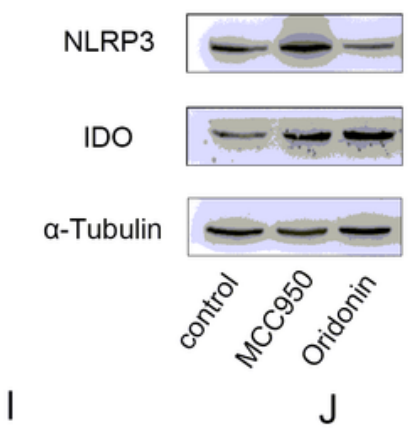

B

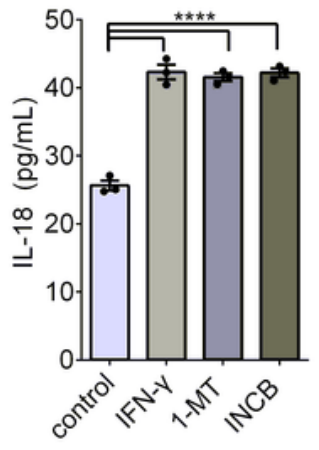

$E$

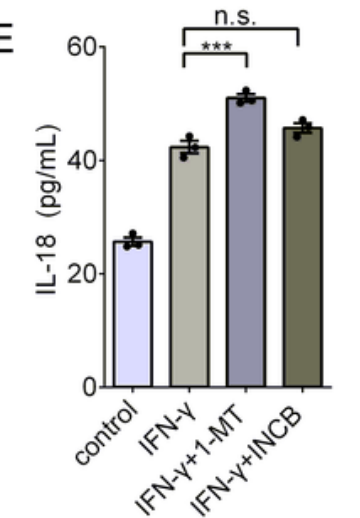

C

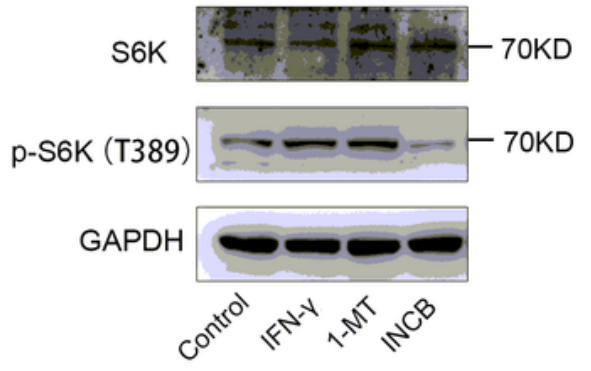

$\mathrm{F}$

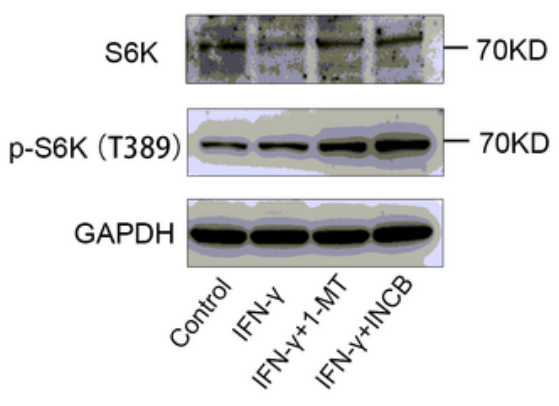

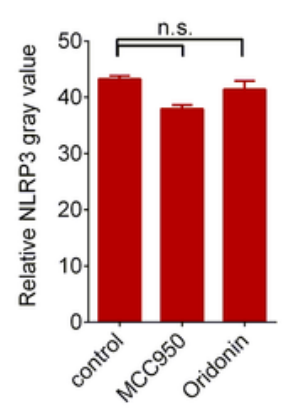

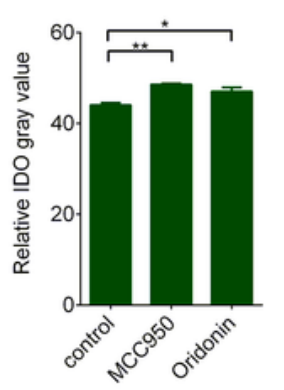

$\mathrm{H}$

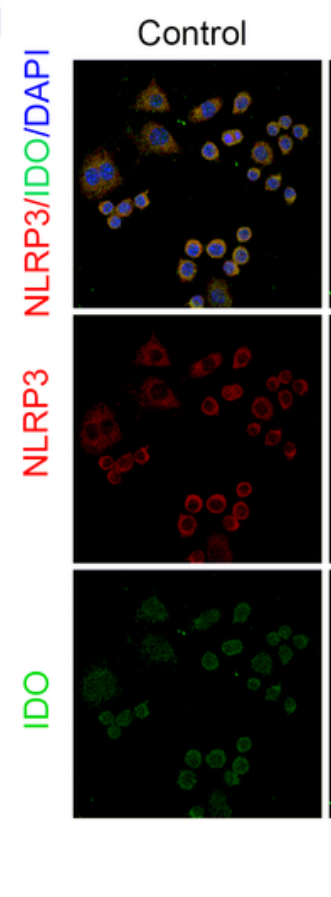

$\operatorname{MCC} 950$
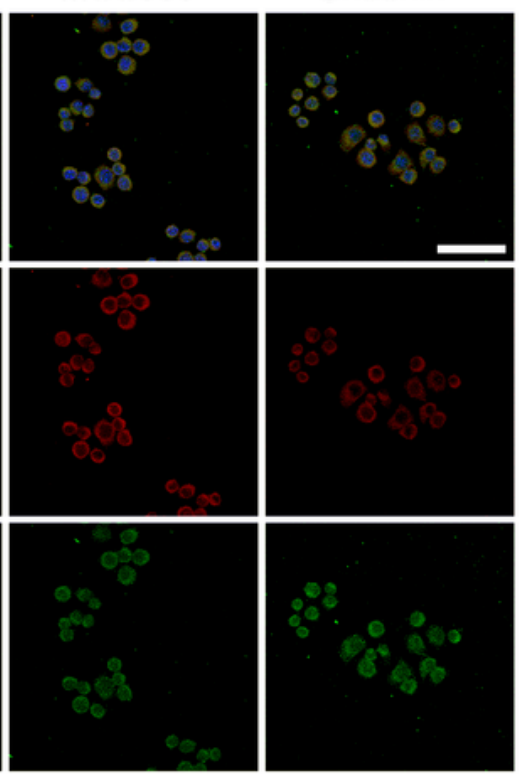

Fig.8

\section{Figure 8}

1-MT and INCB24360 enhance IL-1 $\beta$ secretion in RAW264.7. A, B. IL-1 $\beta$ and IL-18 levels in the culturing medium of RAW264.7 treated by IFN-y, 1-MT and INCB for 24h detected by ELISA. C. S6K and p-S6K protein levels in RAW264.7 treated by IFN- $\gamma, 1-M T$ and INCB for 24h. D, E. IL-1 $\beta$ and IL-18 levels in the culturing medium in RAW264.7 treated by IFN- $\gamma$, IFN- $-+1-M T$ and IFN- $\gamma+$ INCB for 24h. F. S6K and p-S6K protein levels in RAW264.7 treated with IFN- $\gamma$, IFN- $\gamma+1-M T$ and IFN- $\gamma+$ INCB. G. The changes of NLRP3 
and IDO expression in RAW264.7 treated by MCC950 and Oridonin for 24h. H-J. The representative immunostaining results of NLRP3 and IDO RAW264.7 treated with MCC950 and IDO for 24h(H). The relative levels of NLRP3 (I) or IDO (J) intensity in RAW264.7 after drug treatment, measured by image J. Scale bars, $50 \mu \mathrm{m}$. One-way ANOVA; all data are expressed as the mean $\pm \mathrm{SEM}$. ${ }^{*}, \mathrm{P}<0.05, * \star, P<0.01$; ns, no statistical difference.

\section{Supplementary Files}

This is a list of supplementary files associated with this preprint. Click to download.

- Extendeddataofmanuscript.pdf 